\title{
Electromagnetic susceptibility anisotropy and its importance for paramagnetic NMR and optical spectroscopy in lanthanide coordination chemistry
}

Octavia A. Blackburn ${ }^{\mathrm{b}}$, Robert M. Edkins, ${ }^{\mathrm{b}}$ Stephen Faulkner ${ }^{\mathrm{b}}{ }^{*}$, Alan M. Kenwright

${ }^{a}$, David Parker ${ }^{a}$, Nicola J. Rogers ${ }^{\text {a }}$ and Sergey Shuvaev ${ }^{\mathrm{a}}$

a) Department of Chemistry, Durham University, South Road, Durham, DH1 3LE, UK

b) Chemistry Research Laboratory, University of Oxford, 12, Mansfield Road, Oxford, OX1 3TA, UK.

david.parker@dur.ac.uk; stephen.faulkner@chem.ox.ac.uk

The importance of the directional dependence of magnetic susceptibility in magnetic resonance and of electric susceptibility in the optical spectroscopy of lanthanide coordination complexes is assessed. A body of more reliable shift, relaxation and optical emission data is emerging for well-defined isostructural series of complexes, allowing detailed comparative analyses to be undertaken. Such work is highlighting the limitations of the current NMR shift and relaxation theories, as well as emphasising the absence of a compelling theoretical framework to explain optical emission phenomena.

\section{Introduction}

The widespread application of lanthanide ions in diverse fields, from medical imaging and bioassays to the development of magnetic and optical devices, derives wholly from the behaviour of open shell f-electrons. ${ }^{1-3}$ Lanthanide ions have unique optical and magnetic properties that are a direct consequence of their electronic structure. In a magnetic field, the size and directional dependence of the magnetic susceptibility, particularly the relative size of the principal orthogonal components, is of paramount importance in determining the chemical shift and spin relaxation behaviour of nuclei close to the metal centre. ${ }^{4,5}$ In a similar manner, the magnitude and anisotropy of the electric susceptibility tensor defines the nature and magnitude of observed optical transitions, under the constraints imposed by molecular symmetry.

Here, the scope and limitations of current theories that address electromagnetic susceptibility and its anisotropy in lanthanide complexes are assessed. A large body of reliable NMR shift/relaxation and optical emission data is emerging from analyses of well-defined isostructural series of complexes that highlight the limitations of the current theoretical framework. ${ }^{6-9}$ These data give direct insights into the nature of anisotropy at a lanthanide centre, and provide a body of information that can be used to test the effectiveness of theories that strive to rationalise experimental data.

It is already clear that simple 'point charge' approaches are insufficient to deal with observed behaviour. Furthermore, it is evident that treatment of molecular complexes 
cannot neglect the effect of ligand structure and the whole ligand field, and that the use of the simple Russell-Saunders coupling model must be treated with extreme caution. The theories that are used to rationalise experimental data from optical emission and absorption experiments have not had a significant impact on the broader community of f-element scientists. The most well known of these is the Judd-Ofelt theory, ${ }^{10}$ which remains rather intractable and opaque and is rarely applied by practising experimental scientists. Of greater benefit to coordination chemists have been analyses based on consideration of polarisability and symmetry; the ligand polarisation model has arguably been much more useful in guiding progress. ${ }^{11-13}$

There is a need for better theories that can be applied to each domain and allow the experimental scientist to devise new coordination complexes with predictable properties, enabling effective prediction of the major magnetic axes and allowing reliable estimates for the shift and relaxation behaviour of a given nucleus, or the structure and intensity of optical transitions. Such theories need to be able to address the mononuclear complexes treated in this review, before they can be used with any degree of confidence on multinuclear and multi-metallic systems.

\section{Electronic Configuration and Magnetic Susceptibility}

Open shell lanthanide ions can almost always be considered on the basis of occupation of the $4 \mathrm{f}$ orbitals, and the classical treatment of lanthanide-containing systems has been based on the assumption that the $4 \mathrm{f}$ orbitals play little role in bonding. While the optical and magnetic behaviour of most coordination compounds is rationalized on the basis of ground and excited state manifolds that have essentially pure f-orbital character, a few cases, such as $\left[\mathrm{YbCp}_{3}\right]$, have emerged where behaviour can only be rationalised by invoking significant covalency. ${ }^{14}$ However, a simpler approach focused purely on the metal centre has generally been used to rationalize the behaviour of the majority of coordination compounds.

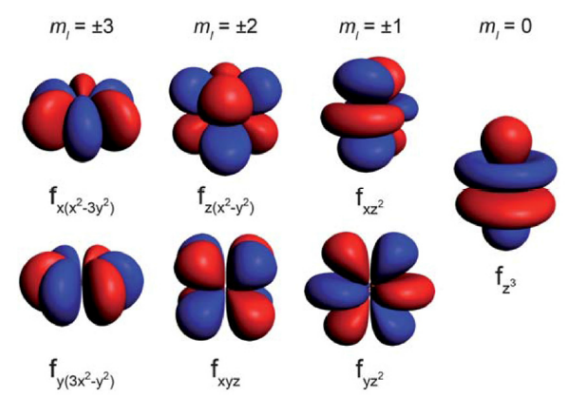

Representations of $4 \mathrm{f}$ orbitals from highest magnitude $m_{l}$ (oblate) to the lowest $m_{l}$ (prolate)

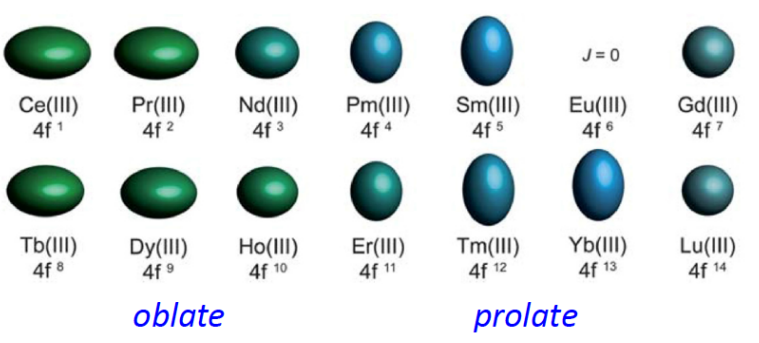

Quadrupole approximations of the $4 \mathrm{f}$ electron distribution, calculated using $J, \alpha\left(2^{\text {nd }}\right.$ order Stevens coefficient) and $\left\langle r^{2}\right\rangle$ (radius of the $4 f$ shell squared).

Figure 1 Shapes of $4 \mathrm{f}$ orbitals and $4 \mathrm{f}$ electron densities; the shape variation of the f electron charge cloud arises from the strong angular dependence of the f orbitals (with permission, adapted from reference 15). 
Historically, the electronic structure of lanthanide ions has been described in terms consistent with free ion behaviour, (Figure 1) using the Russell-Saunders coupling scheme to define the ground and excited states, with term symbols of the form ${ }^{2 S+1} L_{J}$. Spin-orbit coupling is presumed to be much larger than crystal field splitting and so the latter is treated as a small perturbation. The application of a small ligand field to the lanthanide ion results in a loss of degeneracy among the $m_{J}$ states, causing magnetic anisotropy that manifests itself through the spectroscopic and magnetic properties of lanthanide complexes. In any such system, each $m_{J}$ state will have distinctive characteristics that can be defined in terms of $\chi_{x x}, \chi_{y y}$, and $\chi_{z z}$, the axial components of the magnetic susceptibility tensor; crucially, the values of these components differ for each $m_{J}$ state in a given system.

In the Landé and van Vleck approximations generally used to treat lanthanide paramagnetism, $J$ is considered to be a good quantum number and its value is assumed to be independent of the ligand field. ${ }^{16}$ However, such approximations are only valid if the ligand field splitting is small relative to the spin-orbit coupling. Across the series, the former can take values between 50 and $1500 \mathrm{~cm}^{-1}$, while the latter can typically vary between 600 and $2000 \mathrm{~cm}^{-1}$. When the ligand field splitting is large, ${ }^{17,18}$ the concept of $J$-mixing has been invoked to interpret lanthanide optical emission spectra, notably to rationalise the unusual emission behaviour of certain Eu(III) compounds. ${ }^{19}$ Even where smaller ligand field effects are present, the influence of the ligand field upon anisotropy cannot be neglected. Sievers 20 developed a method to represent the three dimensional anisotropy of an $m_{J}$ state. The ideas were later extended by Long ${ }^{15}$ to generate pictorial representations of the $m_{J}$ states for the lowest $J$ states of the tripositive lanthanide free ions (Figures 1 and 2).

$$
m_{J}=1 / 2
$$
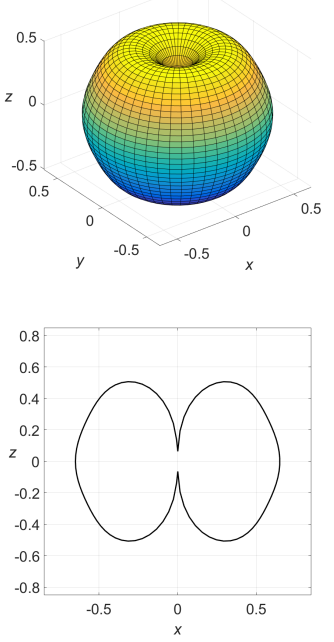

$m_{J}=3 / 2$
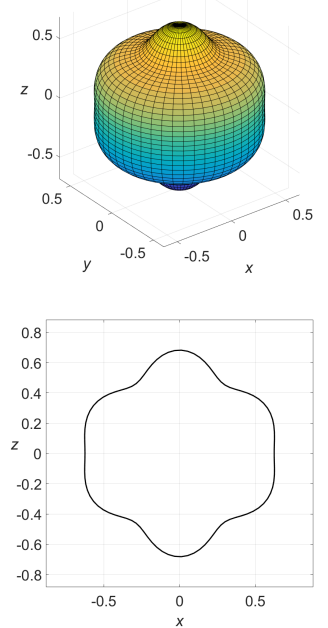

$m_{J}=5 / 2$
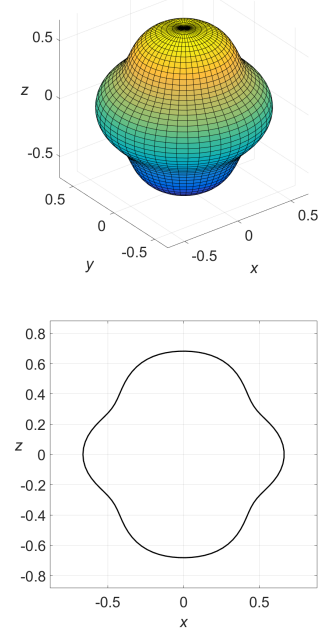

$m_{J}=7 / 2$
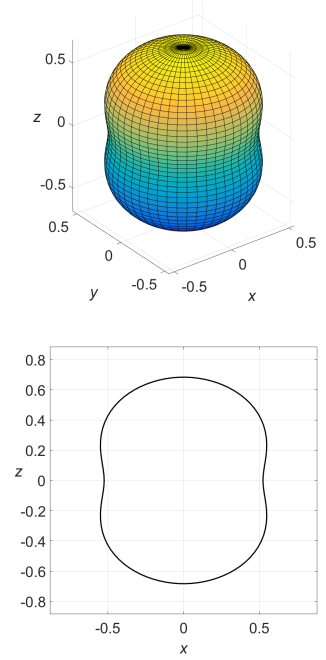

Figure 2 Pictorial representations of the $m_{J}$ states for the ${ }^{2} \mathrm{~F}_{7 / 2}$ state of ytterbium (III), obtained by the method of Sievers ${ }^{20}$ from the hydrogenic 4 f orbital set. 
From these representations, it can be seen that some $m_{J}$ states are prolate (i.e. they align with the magnetic easy axis), while others are oblate (with the principal axis in the easy plane), and we can rationalise how the strength and geometry of the ligand field will influence the energies of these states. At very low temperatures, such as those involved when considering single molecule magnets, it is possible to consider just the lowest energy $m_{J}$ state, and with the aid of low temperature EPR studies $(<10$ $\mathrm{K}$, where $\mathrm{k} T<7 \mathrm{~cm}^{-1}$ ), suitable ligand field models can be described that may provide a lead to improved magnetic properties. ${ }^{21}$

However, the situation is still more complex if we consider spectroscopic techniques that are routinely applied at ambient temperatures. If we consider that the relative energy (and indeed relative order) of the $m_{J}$ states is determined by the crystal field, while the relative population of the $m_{J}$ states will be given by a Boltzmann distribution, it is clear that even subtle changes to the local ligand field can skew the relative populations of the $m_{J}$ manifold. For instance, in computational models of the $m_{J}$ manifold in [DTMA.Yb.F] ${ }^{2+}$, (DTMA:1,4,7,10-tetraazacyclododecane-tetraacetamide) changing the $\mathrm{Yb}-\mathrm{F}$ bond length fundamentally alters the relative energies and populations of the four ytterbium (III) $m_{J}$ levels, $\left({ }^{2} \mathrm{~F}_{7 / 2}\right.$ ground state, with $\pm 7 / 2$, $\pm 5 / 2, \pm 3 / 2$ and $\pm 1 / 2 m_{J}$ states), as illustrated in Figure $3 \mathrm{a}^{8}{ }^{8}$ This change in population can radically alter the nature of the anisotropy, leading, vide infra, to very large changes in observed spectroscopic phenomena. Similarly, changes to the substitution pattern in a series of [Ln.DTMA] derivatives can cause dramatic variations to the observed magnetic anisotropy, suggesting that even subtle changes to the ligand donor set or solvation sphere can be used to "tune" the anisotropy. It is thus necessary to consider the whole of the local environment around the metal centre and its geometry, rather than just the metal itself or the donor set of a multi-dentate ligand.

a)

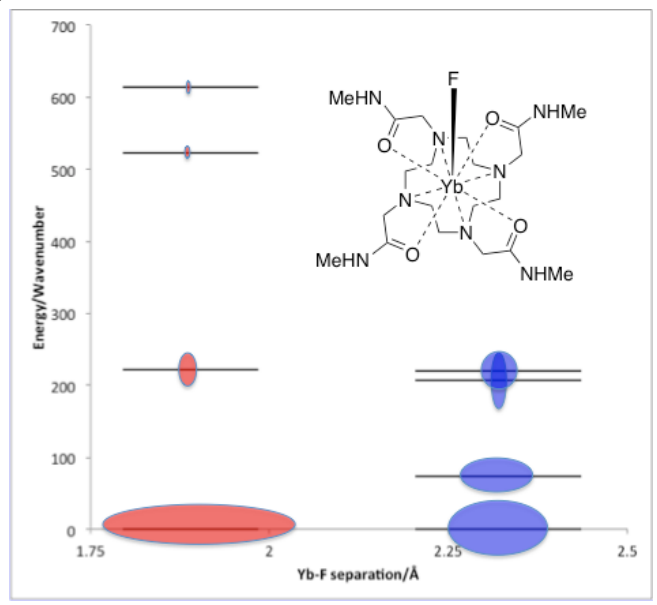

b)

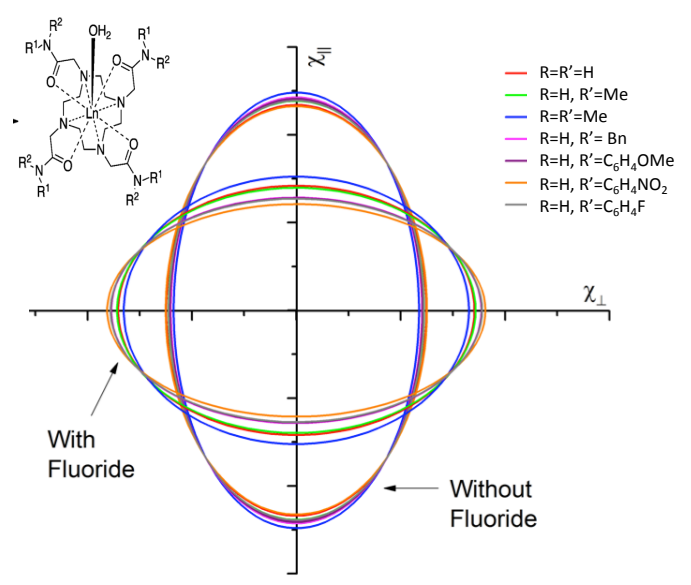

Figure 3 a) Calculated variations in $m_{J}$ energy levels and magnetic anisotropy for [Yb.DTMA.F] ${ }^{2+}$ for Yb-F separations of 1.89 (left) and 2.32 A. The coloured shapes represent vertical sections through a magnetic anisotropy spheroid plane defined by the Yb-F axis; their relative size reflects the Boltzmann population of the levels in each manifold at $298 \mathrm{~K} .{ }^{8} \mathrm{~b}$ ) Representing the variation in observed magnetic anisotropy for a series of ytterbium (III) complexes with ligands related to DTMA, in the presence and absence of fluoride ${ }^{9}$ 
Other factors, particularly symmetry, can influence the nature of the anisotropy at the metal centre. While a spheroid adequately describes the nature of the anisotropy in systems with a high order axis of rotation, an ellipsoid is required to explain the anisotropy in systems with lower symmetry- i.e. three components $\left(\chi_{x x}, \chi_{y y}\right.$ and $\left.\chi_{z z}\right)$ are required. Here again, temperature is important. At low temperatures, coordination of water to the axial site on $\left[\operatorname{Ln} . D O T A\left(\mathrm{H}_{2} \mathrm{O}\right)\right]^{-}$(DOTA: 1,4,7,10tetraazacyclododecane-tetraacetate) or $[\operatorname{Ln} . \operatorname{DTMA}(\mathrm{X})]^{3+}$ complexes lowers the local symmetry at the lanthanide from $\mathrm{C}_{4}$ to an approximation of $\mathrm{C}_{2}$. As a consequence, there is anisotropy in $\chi_{x x}$ and $\chi_{y y}$ as well as in $\chi_{z z}$, meaning that a principal magnetic axis can be readily defined in the easy plane for an aqueous complex (unlike for fluoride complexes, where $\mathrm{C}_{4}$ symmetry persists).

Such phenomena have been observed by Sessoli and co-workers through lowtemperature SQUID measurements ${ }^{22}$, on $\left[\operatorname{Ln} . \operatorname{DOTA}\left(\mathrm{H}_{2} \mathrm{O}\right)\right]^{-}$and also in low temperature calculations on [Yb.DTMA. $\left.\left(\mathrm{OH}_{2}\right)\right]^{3+} \cdot{ }^{8,9}$ At ambient temperatures, rapid rotation about the water-lanthanide axis leads to averaging of $\chi_{x x}$ and $\chi_{y y}$, and so apparent $\mathrm{C}_{4}$ symmetry can be preserved. Sessoli's low temperature work ${ }^{22}$ shows that the direction of the principal magnetic axis in $\left[\operatorname{Ln} \text {.DOTA }\left(\mathrm{H}_{2} \mathrm{O}\right)\right]^{-}$complexes varies with the nature of the lanthanide ion (Figure 4), and also is sensitive to the presence or absence of a coordinated water molecule.

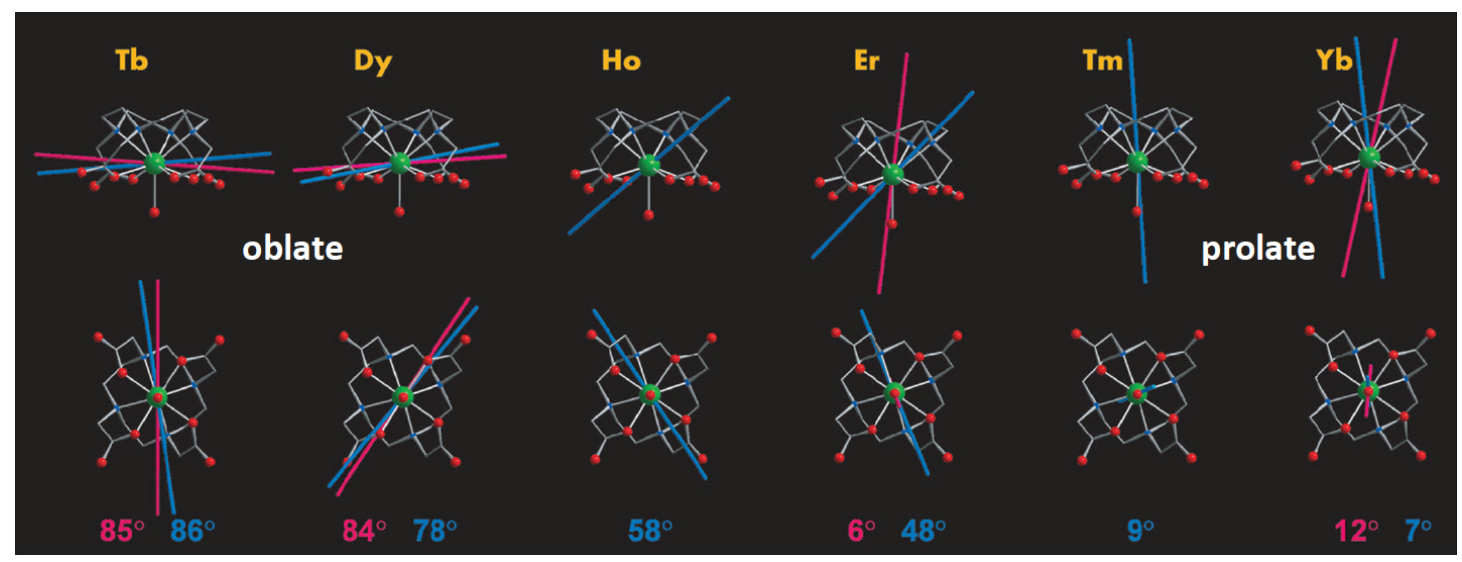

Figure 4 Experimental (pink) and calculated (blue) magnetization easy axis viewed perpendicular (upper) and parallel (lower) to the symmetry axis of [Ln.DOTA( $\left.\left.\mathrm{H}_{2} \mathrm{O}\right)\right]^{-}(2 \mathrm{~K}) .{ }^{22}$ (with permission)

Such behaviour reflects the general finding that the magnetic anisotropy of a paramagnetic lanthanide ion is much more variable than had been originally assumed. While the magnetic anisotropy of lanthanide compounds can readily be inferred from a body of spectroscopic evidence, the absolute magnetic susceptibility, and thus the magnetic moment, requires careful magnetic susceptibility measurements to be undertaken for each complex. The general assumption of a room temperature magnetic moment derived simply from $J$ and the Landé factor $g_{J}$ is not valid. For example, lanthanide oxy-halides have strong ligand fields with second order ligand field parameters of around $-1300 \mathrm{~cm}^{-1}$ (Table 1) ${ }^{23}$ : SQUID magnetometry 
measurements give room temperature magnetic moments, $\mu_{\text {eff }}$, that are all lower than the "free ion" values, notably for Ho $(-12 \%)$. To quote Holsa, ${ }^{23}$ " the effect of the crystal (ligand) field is thus not negligible ... when the room temperature magnetic moment is considered."

Such issues are also likely to influence the magnetic properties of molecular complexes- with significant deviations from predicted values expected to be the norm. SQUID measurements on such systems require careful measurement, paying attention to sample homogeneity and degree of solvation. Regardless of the absolute magnitude of $\chi$, an understanding of the anisotropy can provide the tools to analyse the spectral behaviour of molecular lanthanide complexes.

Table 1. Crystal field values and powder magnetic moments in LnOBr with tetragonal ( $\mathrm{P} 4 / \mathrm{nmm}$ or $\left.\mathrm{C}_{4 \mathrm{v}}\right)$ point symmetry. ${ }^{23}$

\begin{tabular}{lllll}
\hline $\mathrm{Ln}$ & $B_{0}^{2} / \mathrm{cm}^{-1}$ & $B_{0}^{4} / \mathrm{cm}^{-1}$ & $\mu_{\mathrm{eff}}(\mathrm{obs})$ & $\mu_{\mathrm{eff}}(\mathrm{calc})$ \\
\hline $\mathrm{Tb}$ & -1199 & -942 & 9.63 & 9.71 \\
$\mathrm{Dy}$ & -1229 & -971 & 10.25 & 10.61 \\
$\mathrm{Ho}$ & -1259 & -1000 & 9.39 & 10.56 \\
$\mathrm{Er}$ & -1289 & -1030 & 9.01 & 9.57 \\
$\mathrm{Tm}$ & -1319 & -1059 & 7.17 & 7.57 \\
$\mathrm{Yb}$ & -1349 & -1088 & 4.41 & 4.55
\end{tabular}

Critical assessment of Bleaney's theory of magnetic anisotropy and recent approaches

The current understanding of the effects of magnetic anisotropy on the observed NMR shifts in a lanthanide complex can be traced back to a theory developed by Bleaney, over 40 years ago. ${ }^{24}$ Although there has been discussion of the limits of its applicability, ${ }^{25-30}$ it remains the basis on which most of the NMR shifts in paramagnetic complexes are interpreted.

In the last 10 years or so, full quantum mechanical treatments have been considered, notably by Autsbach, ${ }^{25}$ showing how NMR chemical shifts can be calculated for paramagnetic complexes from first principles. In order to circumvent the limitations in DFT theory arising from the use of common but approximate functionals, an $a b$ initio method has been promulgated to treat open-shell paramagnetic systems, in combination with a theoretical formulation for paramagnetic NMR shielding. The approach also avoids the difficulties associated with the use of calculated EPR parameters or magnetic susceptibilities. It relies upon recent advances made by Soncini ${ }^{26}$ in which the NMR shielding tensor is considered as a temperaturedependent derivative of the total free energy, in which the shielding tensor elements of a paramagnetic system are given by a sum-over-states expression. ${ }^{25}$ This treatment clearly works well with simple inorganic complexes, but is likely to exhibit steep 
complexity scaling as the size of the complex increases, particularly for strongly correlated systems. It represents a very promising approach a priori; although in an ideal world, a theoretical treatment would still need to include dynamic electron correlation and take account of spin polarization.

An excellent presentation of Bleaney's theory, together with some discussion of its limitations is contained in the work of Piguet and Geraldes. ${ }^{4}$ Briefly, the theory states that the magnetic susceptibility anisotropy is responsible for the pseudocontact shifts (PCS) in the NMR spectrum of a lanthanide (III) complex. The contact shift involves through-bond transmission of electron density from the lanthanide to the nucleus under inspection and therefore falls off rapidly with the number of intervening bonds, and is generally assumed to be negligible beyond four bonds. According to Bleaney's theory, the pseudocontact shifts are described in equations (1) and (2) below,

$$
\begin{aligned}
\delta_{\text {pseudo }} & =\frac{C_{J} \mu_{B}^{2}}{60(k T)^{2}}\left[\frac{\left(3 \cos ^{2} \theta-1\right)}{r^{3}} B_{0}^{2}+\frac{\left(\sin ^{2} \theta \cos 2 \varphi\right)}{r^{3}} B_{2}^{2}\right] \\
C_{J} & =g_{J}^{2}\langle J\|\alpha\| J\rangle J(J+1)(2 J-1)(2 J+3)
\end{aligned}
$$

where $\theta, \varphi$, and $r$ define the polar coordinates and internuclear distance to the lanthanide(III) ion, $C_{\mathrm{J}}$ is the Bleaney constant, $\mu_{\mathrm{B}}$ is the Bohr magneton, $B_{0}^{2}$ and $B_{2}^{2}$ are second order ligand field splitting parameters, $\langle J\|\alpha\| J\rangle$ is a numerical coefficient, $J$ is the total angular momentum and $g$ the electron g-factor. Equation (1) can also be expressed in terms of the size of the orthogonal magnetic susceptibility components, eq. (3), removing some uncertainty inherent in ligand field term descriptors:

$\delta_{\text {pseudo }}=\frac{1}{2 N}\left[\frac{\left(3 \cos ^{2} \theta-1\right)}{r^{3}}\left(\chi_{z z}-\chi_{a v}\right)+\frac{\left(\sin ^{2} \theta \cos 2 \varphi\right)}{r^{3}}\left(\chi_{x x}-\chi_{y y}\right)\right]$

The Bleaney constant varies with the nature of the lanthanide(III) ion, but is considered to be a property of the lanthanide only, i.e. it is independent of the ligand. Values of the Bleaney constants for 'free' lanthanide ions are tabulated in the literature. ${ }^{24,31}$

While Bleaney's theory has been found to conform to experimental observations in a number of systems, it contains assumptions and approximations that limit its applicability. First, it assumes that the magnetic effects of the unpaired electrons on the lanthanide can be approximated by a point dipole on the lanthanide nucleus. While there is no theoretical justification for this, it was probably a reasonable approximation at the time because no better tools existed for dealing with the problem. This situation has recently changed; in 2014, Charnock and Kuprov described a particularly elegant mathematical procedure to model the effects on observed NMR shifts of unpaired electrons whose positions are described by a nonpoint electron probability density. ${ }^{32}$ Their approach uses classical physics, rooted in 
Maxwell's equations; it is intrinsically directly applicable to paramagnetic systems and should, in principle, improve the quality of shift prediction.

Second, Bleaney theory assumes that the ligand field parameters, $B_{0}^{2}$ and $B_{2}^{2}$ are small compared to $\mathrm{k} T$. If this condition is fulfilled, $J$ is a good quantum number and accurately describes the effects of spin-orbit coupling on the system. However, if this condition is not fulfilled and the $2 J+1$ splittings due to the crystal field parameters are of the same order as or larger than $\mathrm{k} T$, the result is overlap of different $J$ bands leading to the phenomenon of $J$ mixing, in which case $J$ is no longer a good quantum number. ${ }^{17}$ The impact can be appreciated by considering eq. (2), above. At $298 \mathrm{~K}$, $\mathrm{k} T$ is about $205 \mathrm{~cm}^{-1}$. For simple lanthanide salts in crystal form (the case Bleaney was working on), the relevant crystal field splitting parameters may well be only tens of wavenumbers, but for lanthanide ions encapsulated in multidentate ligands in solution at room temperature, the relevant ligand field splittings are often considerably greater than $205 \mathrm{~cm}^{-1}$. It is not trivial to measure every ligand field splitting parameter directly. However, a good indication of the size of the $B_{0}^{2}$ term in axially symmetric complexes can be derived from the splitting of the $\Delta J=1$ band in the $\mathrm{Eu}^{3+}$ emission spectrum, where the true value of $B_{0}^{2}$ is directly proportional to the observed splitting. ${ }^{18,33}$ Even here, it is necessary to add the caveat that the natural linewidth in such spectra can inhibit the determination of $B_{0}^{2}$ in systems where the splitting is small, unless enhanced resolution is available through analysis of circularly polarized emission spectra. The constant of proportionality is believed to lie between 3.3 and 4.1 , but even taking the lower limit, it is easy to demonstrate that many complexes violate Bleaney's assumption, and magnitudes of $B_{0}^{2}$ in excess of $1500 \mathrm{~cm}^{-1}$ have been reported. ${ }^{18}$ This situation has been noted earlier, ${ }^{4,30}$ yet the widespread violation of this assumption is often still ignored.

Third, Bleaney's approach assumes that only the $B_{0}^{2}$ and $B_{2}^{2}$ crystal field terms need to be considered, because the effect of higher order terms is so small as to be negligible. Again, while this is probably a reasonable approximation for the systems Bleaney was working on, its general validity is by no means assured, especially since the higher order crystal field terms are more difficult to measure so we often have no idea of their actual size. The assumption that only the lower order terms need to be considered derives from the $T^{2}$ variation of chemical shift. ${ }^{1}$ However, the temperature range over which NMR shifts can be measured in solution is often relatively narrow, so it is hard to exclude all possible effects on that basis alone. In systems where higher order crystal field terms have been determined, it has been shown that they are often of the same order of magnitude as the lower order terms ${ }^{23}$ and therefore may contribute to the observed shift to an extent not accounted for by Bleaney's theory.

However, it is worth noting that the shift dependence on higher order terms will vary with $1 / T^{\geq 3}$ and so will drop off quickly with increasing temperature. Furthermore, 
values of $B_{q}^{k}$ scale as $r^{-(\mathrm{k}+1)}$, where $r$ is the ligand-metal distance, and therefore would be expected to decrease with increasing $\mathrm{k}$. The non-zero terms are restricted both by $J$ ( $\mathrm{k} \leq 2 \mathrm{~J}$ and $|\mathrm{q}| \leq \mathrm{k}$ ), and by the symmetry around the lanthanide, with many more terms being relevant for higher $J$ systems and when the symmetry is lowered.

A number of attempts have been made to estimate the contribution of higher order terms to the lanthanide-induced shift. Several early publications suggested that the accuracy gained by a more comprehensive theory would not compensate for the complicated mathematics involved. McGarvey's estimation of the $T^{3}$ term implied that Bleaney's theory should be accurate to within $10-20 \%$, based on previously determined crystal field parameters of lanthanide-containing crystals. ${ }^{25}$ Golding and Pyykkö also estimated deviations from Bleaney theory of less than $20 \%$ when higher crystal field components were added in $\mathrm{D}_{3 \mathrm{~h}}$ or $\mathrm{C}_{3 \mathrm{~h}}$ symmetry. ${ }^{28}$ However, Binnemans later contested the validity of these studies. Lanthanide compounds were examined with only small ligand field splitting energies compared to $\mathrm{k} T,{ }^{29,30}$ and it was asserted that for compounds with large ligand field splittings the high temperature expansion of the magnetic susceptibility used by Bleaney is inadequate. Rigorous numerical calculations of the magnetic anisotropy of model systems were largely in disagreement with Bleaney's theory, with the ratios of anisotropies for different lanthanide ions being sensitive to the coordination geometry, as opposed to being fixed according to $C_{J}$. The sequence of anisotropies across the series was particularly badly accounted for when $B_{0}^{2}$ values were small. Terbium and thulium ions were calculated to have the largest magnetic anisotropies of the series in contrast to Bleaney's prediction that dysprosium should always be the maximum. The exclusion of higher order parameters was found to be inaccurate using either approach, although the variance of the sign of the magnetic anisotropy predicted by Bleaney was found to hold in most circumstances.

Evaluating how well Bleaney's theory works for real systems is non-trivial, firstly because the ligand field parameters are usually not known and secondly because the number of systems for which the NMR assignments can be made from first principles is limited. In practice, the theory often provides a model against which NMR peaks are assigned. Indeed, the primary application of Bleaney's theory is in structural biology where fitting of observed NMR shifts to the Bleaney model for a protein labelled with a lanthanide tag provides additional distance constraints for molecular geometry determination. ${ }^{34}$ This approach does not tell us how well the Bleaney model works, particularly for nuclei that are relatively close to the lanthanide, the case where the point dipole approximation is least satisfactory and the contact shift is most obstructive. Further, observations on a single complex are a poor test of Bleaney's model because, while a plot of PCS against the geometrical factors in equation (1) may give a straight line, this is not proof that the values for the Bleaney constant and the ligand field terms are appropriate. A more stringent evaluation of the theory can be made when looking at the data for a number of different lanthanides across an 
isostructural series of complexes. In this case, it is assumed that the ligand field terms would stay more or less constant across the series, although this assumption has been questioned, ${ }^{4}$ so that the variation in shift for a given resonance should be proportional to the Bleaney constants. It must be noted that several authors have implied that a variation in the crystal field parameter across the series is responsible for anomalous behaviour, although the physical basis for the putative variation is not apparent. ${ }^{35-38}$

Several isostructural series have recently been investigated. ${ }^{6}$ It was found that the correlation between the PCS and the relevant Bleaney constants for a particular resonance more than $5 \AA$ distant from the lanthanide (where the contact shift can be neglected) was poor, especially for systems lacking a $\mathrm{C}_{3}$ or $\mathrm{C}_{4}$ time-averaged symmetry axis, (Figure 5). Note that one example has axial symmetry but the other does not. In each case, the isostructurality of the series and the absence of a significant contact contribution was confirmed by assessment of Reilley plots. ${ }^{38}$

The implication of such behaviour is that either the ligand field term is influenced by the electronic structure of the lanthanide and the Bleaney constants tabulated for free lanthanide ions are not applicable to all complexes, or that the variation in shift across the series is a more complex function of lanthanide environment and is not fully accounted for in the original theory. Further discrepancies become apparent when looking at the results across structurally related series of complexes. The shifts for aryl resonances of three related isostructural series based on the trispyridyltriazacyclononane motif (Figure 6) also deviate from Bleaney behaviour. ${ }^{6}$ In these cases, notwithstanding any contact shift contribution, it would be expected that the order of the shifts for each series would be determined by the Bleaney constants, and that the magnitudes of the shifts would scale with the crystal field terms. For each series it was possible to estimate $B_{0}^{2}$ from the splitting of the $\Delta J=1$ band in the Eu emission spectrum. It is clear that while the trends for some lanthanides approximately follow the size of $B_{0}^{2}$, others do not. In this particular case, the shifts for the Dy and Er complexes deviate markedly. The Reilley plots of the same resonances emphasize that the shifts of these lanthanide complexes do not follow the expected order according to $C_{J} /<S_{Z}>$. In particular, thulium has often been noted as an anomaly in such treatments. ${ }^{39-41}$ 

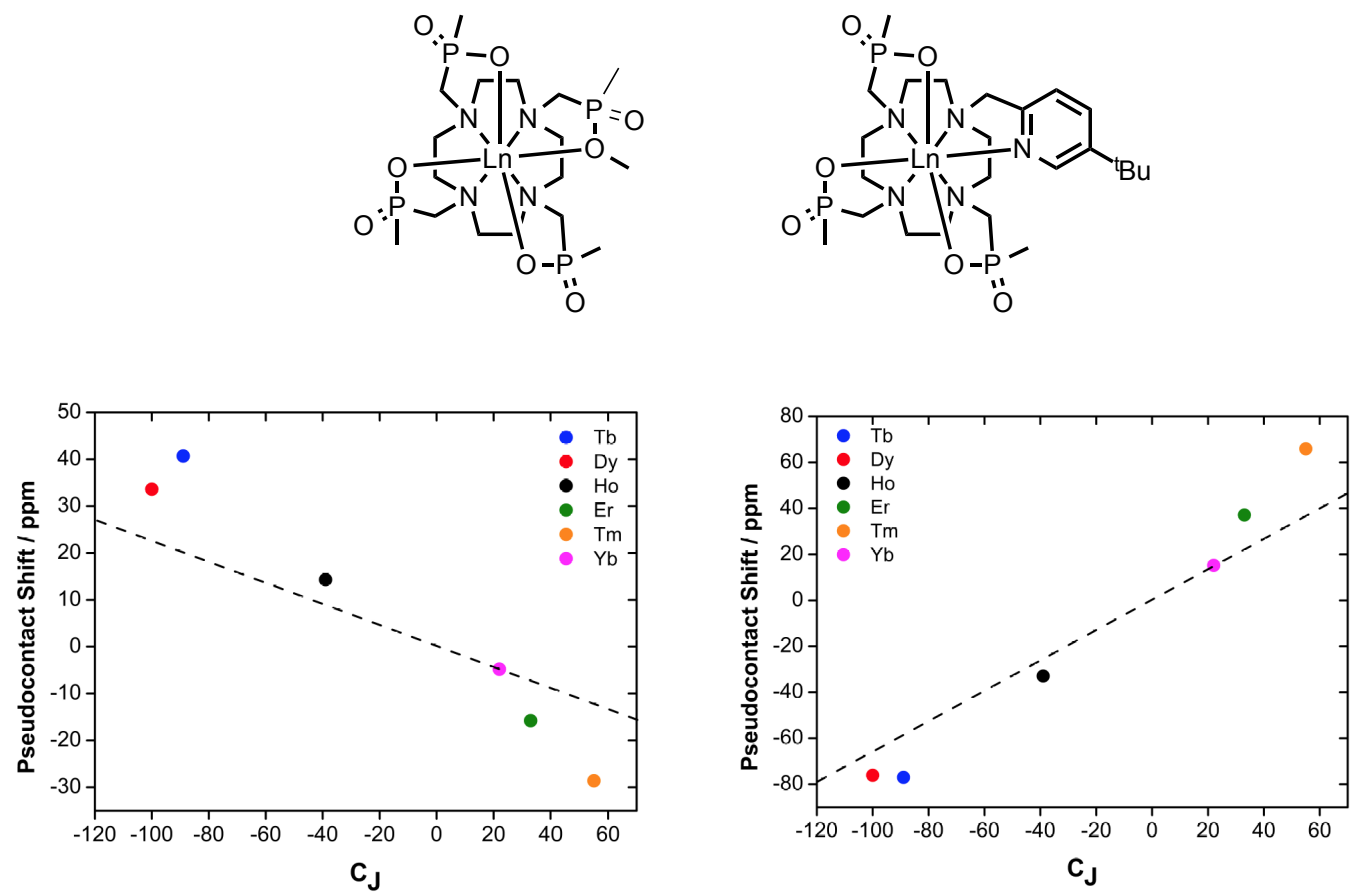

Figure 5 Plots of paramagnetic shifts $v s$ Bleaney constants for two eight-coordinate isostructural series; (left) methyl signal; (right) $t$-butyl signal (295K).

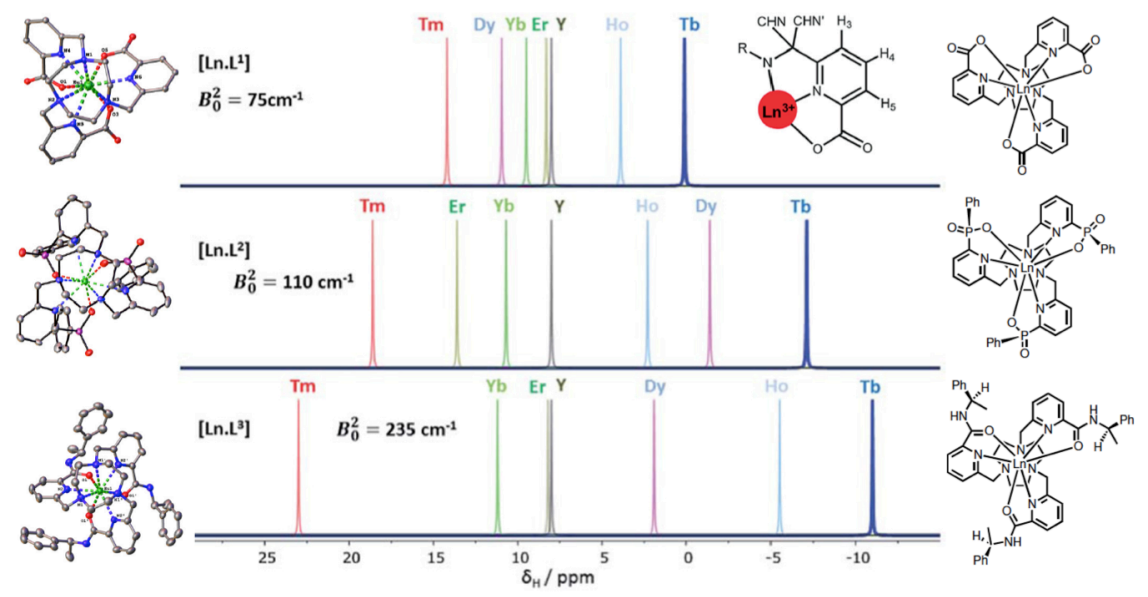

Figure 6 Observed shifts of picolyl $\mathrm{H}_{3}$ ring resonances across three isostructural series. Similar behaviour was observed for $\mathrm{H}_{4}$ and $\mathrm{H}_{5} .{ }^{6}$

The limitations of Bleaney's theory have already been pointed out, but there are some additional factors that may also limit the applicability of the theory which are less well known. The first of these is the assumption that the direction of the principal magnetic axis is fixed by the ligand and, for an axially symmetric ligand, will generally be coincident with the symmetry axis. As discussed above, Sessoli's work shows that this is not necessarily the case. ${ }^{22}$ The direction of the principal magnetic axis can vary with the nature of the lanthanide ion (Figure 4, vide supra). 
Another limitation is the fact that the point dipole approximation is clearly inaccurate. Rinehart and Long have recently shown ${ }^{15}$ that the electron density clouds for the maximum $\left|m_{J}\right|$ projections are normally prolate for $\mathrm{Yb}, \mathrm{Tm}, \mathrm{Er}, \mathrm{Sm}$, and $\mathrm{Eu}$ (when the population of low lying excited states is taken into account), and are usually oblate for $\mathrm{Ce}, \mathrm{Tb}, \mathrm{Pr}, \mathrm{Dy}, \mathrm{Nd}$, and Ho. Moreover, the details of the electron distribution are sensitive to the ligand field (Figure 2). The approach has considerable merit, in that Long identifies a fundamental issue- namely that the whole crystal field defines the nature of the electron distribution. Hence, ligand electron density on the molecular $z$ axis should destabilise maximum $\left|m_{J}\right|$ for the prolate ions and stabilise them for the oblate. The f-electron density distribution is then a rather complex function of the strength and geometry of the ligand field. The recent, pioneering work of Yamashita and Enders has already begun to address detailed NMR analyses of complexes whose magnetic susceptibility behaviour has been rigorously established. Their approach offers a glimpse of what can be done, for example, in the single molecule magnet systems based on terbium phthalocyanato complexes. 15

It has long been known that in 9-coordinate systems the pseudo-contact shift is exquisitely sensitive to the nature of the axial ligand in numerous mono-capped square-antiprismatic systems. ${ }^{42,43}$ More recently ${ }^{8,9}$ it has been shown that substitution of water with fluoride as the axial ligand in such a system results in profound changes to the spectroscopic properties of the complex. These observations were interpreted on the basis that the balance of the equatorial ligand field of the eight-coordinate ligand and the axial field from water coordination will stabilize the prolate $m_{J}= \pm 7 / 2$ states of the ${ }^{2} \mathrm{~F}_{7 / 2}$ ion. Coordination of anionic fluoride on the four-fold axis in place of water generates a dominant axial field that stabilizes the oblate $m_{J}= \pm 1 / 2$ states. This results in a change from easy-axis $\left(\chi_{\|}>\chi_{\perp}\right)$ to easy-plane $\left(\chi_{\|}<\chi_{\perp}\right)$ magnetic anisotropy, which in turn reverses the peak order in the proton NMR spectrum of the $\mathrm{Yb}^{3+}$ complex (Figure 7).

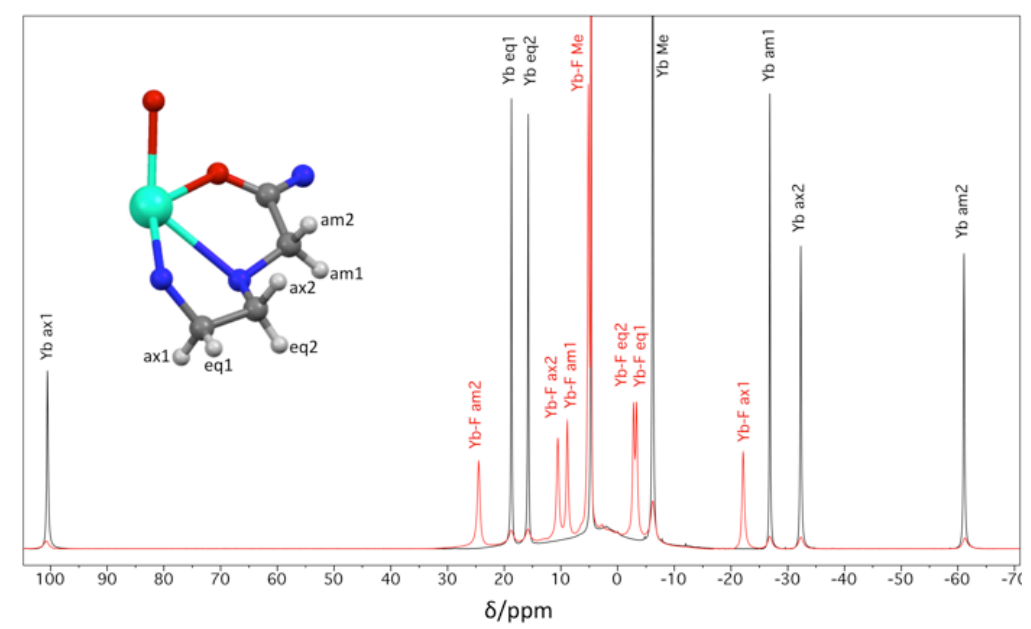


Figure $7{ }^{1} \mathrm{H}$ NMR spectra $\left(400 \mathrm{MHz}, 298 \mathrm{~K}, \mathrm{D}_{2} \mathrm{O}\right)$ of $[\mathrm{Yb} . \mathrm{DTMA}]^{3+}\left[(\mathrm{OTf})_{3}\right]^{3-}$ in the absence (black) and presence (red) of an excess of sodium fluoride, with assignments based on the labelled quadrant of the complex.

Although the observed chemical shifts are proportional to the geometric factor (eq. 1) in each case, the size of the mutually orthogonal components of the magnetic susceptibility tensor are highly dependent on the crystal field. In the case of fluoride substitution, the position of the principal axis effectively switches, as may occur with lanthanide ion permutation in a common complex. ${ }^{12}$ It is therefore clear that the magnetic anisotropy relies on a subtle interplay between the ground state electronic distribution of the lanthanide ion and the ligand field as appropriate. The higher order ligand field terms effectively define the finer details of the electronic distribution, so the extent of their effect on the observed shifts will depend on both the lanthanide ion and the ligand field under scrutiny and hence should be included in a thorough treatment.

It is evident that Bleaney's description of the magnetic anisotropy in lanthanide ions contains a number of assumptions and approximations that result in it failing to fully represent the observed behaviour in a significant number of cases. In some instances, this failure only becomes apparent when viewing the behaviour of the whole isostructural series. Moreover, recent work shows that the actual behaviour observed relies on the subtle interplay between the anisotropic distribution of $4 \mathrm{f}$ electron density on the lanthanide (not point dipole) and the polarity (and polarisability) of the ligand field, which can in turn affect the structure of the ground state $m_{J}$ manifold. The separation of this interplay into a ligand field descriptor, believed to be independent of the lanthanide in question and a (known) Bleaney constant based on the assumption that $J$ is a good quantum number, is inadequate.

\section{Spin Relaxation Theory and Electronic Relaxation}

The role of longitudinal and transverse relaxation for ligand nuclei in lanthanide complexes is an important issue in the design of molecular probes for MRI applications, both for the direct detection of paramagnetic agents ${ }^{6,44}$ and PARACEST agents. ${ }^{45}$ In the former case, enhanced relaxation rates allow fast acquisition times; in the latter, the nuclear relaxation rate must be slow enough to allow efficient saturation transfer for CEST detection. The mere presence of electron spin within a paramagnetic system enhances the rate at which nuclear spins relax to thermal equilibrium, following saturation with a radiofrequency pulse. The electronic magnetic momentum of the unpaired electrons within the lanthanide ion generates fluctuating magnetic fields that can induce nuclear spin transitions for nuclei whose magnetic momentum can interact with that of the electrons. These fluctuations enhance nuclear relaxation. 
The nucleus of interest experiences the spin density of the unpaired electrons, distributed over space, and the relaxation enhancement occurs via three different mechanisms of electron-nuclear relaxation. First, fluctuating electron spin density located at the resonating nucleus gives rise to the through-bond contact relaxation enhancement (also called hyperfine or scalar contribution), although this contribution to the overall relaxation is deemed negligible for $\mathrm{Ln}^{3+} \neq \mathrm{Gd}^{3+} .{ }^{46}$ Second, fluctuation of the remaining electron density of the molecule is felt through space by the nucleus, and gives rise to dipolar coupling, and third, the time-averaged static electron spin, due to the small population difference in spin energy levels, is also felt through space, and gives rise to the Curie contribution, due to fluctuations on the slower time-scale of molecular rotation. ${ }^{4,5}$

Current spin relaxation theory of lanthanide complexes stems from either perturbative treatments using Bloch-Redfield Wangsness (BRW) theory ${ }^{47}$ or more general, nonperturbative approaches, based on the stochastic Liouville method. ${ }^{48}$ The latter approach suffers in practice from very large matrix dimensions, and is limited to very small spin systems. However, many of the assumptions made when using BRW theory are violated for the unpaired electrons in paramagnetic systems. Such a perturbative treatment requires consideration of a large number of empirical parameters that are difficult to quantify for complex systems (vide infra), although large data sets can be fitted using global minimizations to estimate such values. ${ }^{49,50}$

The Solomon-Bloembergen-Morgan (SBM) equation (eq. 4), ${ }^{51-54}$ derived from BRW theory, is a summation of the dipolar and Curie contributions, and is most commonly employed to describe intramolecular longitudinal relaxation rates in $\mathrm{Ln}^{3+}$ coordination complexes. It assumes isotropic magnetic susceptibility (vide infra),

$\frac{1}{T_{1}}=\frac{2}{15}\left(\frac{\mu_{0}}{4 \pi}\right)^{2} \frac{\gamma_{N}^{2} \mu_{e f f}^{2}}{r^{6}}\left[\frac{7 \tau_{c}}{1+\omega_{e}^{2} \tau_{c}^{2}}+\frac{3 \tau_{c}}{1+\omega_{N}^{2} \tau_{c}^{2}}\right]+\frac{2}{5}\left(\frac{\mu_{0}}{4 \pi}\right)^{2} \frac{\omega_{N}^{2} \mu_{e f f}^{4}}{\left(3 k_{B} T\right)^{2} r^{6}}\left[\frac{3 \tau_{r}}{1+\omega_{N}^{2} \tau_{r}^{2}}\right]$

where $\mu_{0}$ is the vacuum permeability, $\gamma_{N}$ is the gyromagnetic ratio of the nucleus of interest, $r$ is the electron-nuclear distance, $\tau_{r}$ is the rotational correlation time of the molecule, $\omega_{N}$ is the nuclear Larmor frequency, $\omega_{e}$ is the electron Larmor frequency, $\mu_{\text {eff }}$ is the effective magnetic moment of the coordinated lanthanide ion, $T$ is the temperature, $\mathrm{k}_{\mathrm{B}}$ is the Boltzmann constant, and $\tau_{c}$ is the stochastic correlation time of the dipolar electron-nuclear modulation. This equation only applies within the limit that the energy of the coupling between the nucleus and the electrons that is being modulated to give rise to spin relaxation, is a lot smaller than $\tau_{c}^{-1}$ itself - this is called the Redfield limit, and can be expressed as $T_{l}^{-1}<<\tau_{c}^{-1}$.

The modulation of the dipolar electron-nuclear interaction leads to a stochastic correlation time, $\tau_{c}$, which in the absence of chemical exchange, can be related to the rotational correlation time, $\tau_{r}$, (usually $c a \cdot 10^{-10} \mathrm{~s}$ ), and an electron spin relaxation time, denoted $T_{I E}$. The values of $T_{I E}$ tend to be $c a \cdot 10^{-12}$ to $10^{-13} \mathrm{~s}$ for fast relaxing 
$\mathrm{Ln}^{3+}$ complexes $\left(\mathrm{Ln}^{3+} \neq \mathrm{Gd}^{3+}\right)$, and so can often determine $\tau_{c}$. Therefore, electronic relaxation is particularly important when predicting the relaxation properties of $\mathrm{Ln}^{3+}$ complexes, especially at low magnetic field strengths where the dipolar contribution dominates the SBM equation (eq. 4), as seen below (Figure 8). ${ }^{7}$

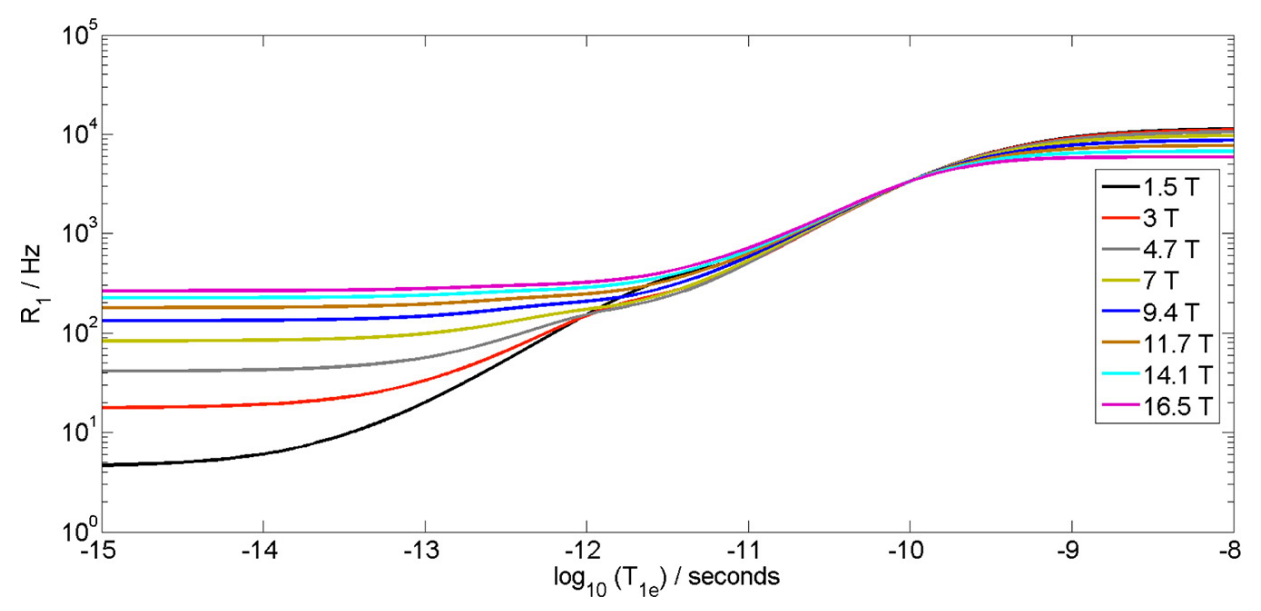

Figure 8 Simulation of the dependence of the ${ }^{19} \mathrm{~F}$ relaxation rate, $\mathrm{R}_{1}$, on electronic relaxation rate at different magnetic field strengths, using the SBM (eq. 4), for a hypothetical $\mathrm{Ln}^{3+}$ complex bearing a $\mathrm{CF}_{3}$ reporter group. $\left(\mu_{\mathrm{eff}}=10 \mathrm{BM}, r=6 \AA, \tau_{\mathrm{r}}=250 \mathrm{ps}, 295 \mathrm{~K} .\right)^{7,50}$

For many $\mathrm{Ln}^{3+}$ complexes, $T_{1 E}$ values fall in the range $10^{-12}-10^{-13} \mathrm{~s}$, which can have an effect of over a factor of 10 on the longitudinal relaxation rate $\left(R_{l}\right)$ at the low magnetic fields where clinical applications of paramagnetic agents are most relevant. The value of $T_{1 E}$ is deemed to be independent of magnetic field, and has only a very small temperature dependence. It increases only slightly with decreasing temperature due to the small activation energy for the electron spin relaxation process. ${ }^{55}$

In 2012, Fries and Belorizky ${ }^{55}$, reiterated what Morgan had earlier suggested, ${ }^{51}$ that $T_{l E}$ can be interpreted as the fluctuation in the static and transient ligand fields because the strong spin-orbit coupling in lanthanide complexes purports that such modulations instigate electronic spin transitions. The modulation of the static ligand field by Brownian rotation of the molecule was thought to have a minor influence on $T_{I E}$ as it gives rise to temporal modulation that is much too slow (hundreds of $\mathrm{ps}$ ) to affect the fast rate of electron relaxation. Therefore, it is believed that the modulation of the transient field lies at the crux of electronic relaxation in solution. The bombardment of a given complex by solvent molecules, with a correlation time in the ca. $10^{-13} \mathrm{~s}$ timescale, gives rise to sudden changes in the rotational motion of the molecule that modulates the orbital magnetic moment. Using this theory ${ }^{10} T_{1 E}$ was initially hypothesized to scale with $|J|$ and vary inversely with the square of the transient ligand field Hamiltonian.

It has been shown, however, that $T_{I E}$ values calculated from experimental data for well-defined series of isostructural $\mathrm{Ln}^{3+}$ complexes, using the SBM equations, follow 
the order $[\mathrm{Tb}, \mathrm{Dy}, \mathrm{Er}]>\mathrm{Ho}>[\mathrm{Tm}, \mathrm{Yb}],{ }^{50}$ Similar trends have been reported by Aime ${ }^{56}$ and Williams. ${ }^{39}$ In each case, the $T_{I E}$ value for the $\mathrm{Ho}^{3+}$ complex $(J=16 / 2)$ in an isostructural series was surprisingly low. The $T_{I E}$ values of highly symmetrical systems appear to show a direct dependence on the second-order static ligand field coefficients, $B_{S, 0}^{2}{ }^{50}$ taken from emission spectral analysis of analogous $\mathrm{Eu}^{3+}$ complexes, observing their $\Delta J=1$ energy level splitting. Therefore, a simple correlation between transient ligand field coefficients, $B_{T, 0}^{k}$, and their static counterparts, $B_{S, 0}^{k}$, for a given complex cannot be used. In addition, the model for collision-induced transient ligand field modulation needs to account for the second and higher order parameters of the ligand fields to determine $T_{I E}$. The sign and magnitude of such parameters may vary greatly from one ligand field to another, and their relative weightings vary with the $\mathrm{Ln}^{3+}$ ion. Hence, the $T_{I E}$ values of isostructural series cannot simply follow $|J|{ }^{50}$

There are several experimental data sets that are difficult to rationalise using BRW theory: ten isostructural series of lanthanide complexes with different local symmetries at different magnetic fields were recently examined. ${ }^{7}$ It was found that the proton relaxation rates of the ${ }^{t} \mathrm{Bu}$ reporter group in octadentate azaphosphinate systems differed markedly from their carboxylate analogues (Figure 9).

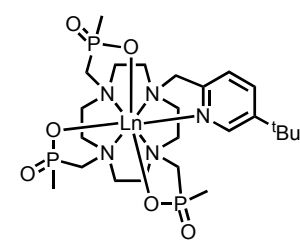

$\left[\right.$ Ln. L $\left.^{1}\right]$

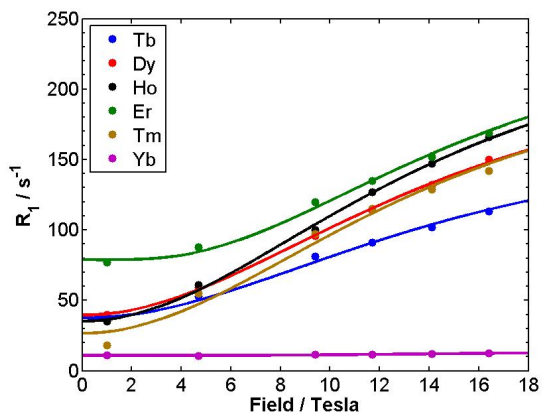

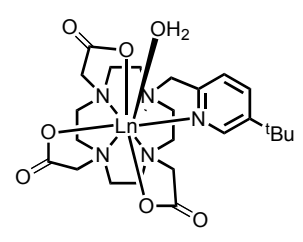

$\left[\operatorname{Ln} \cdot \mathrm{L}^{2}\left(\mathrm{H}_{2} \mathrm{O}\right)\right]$

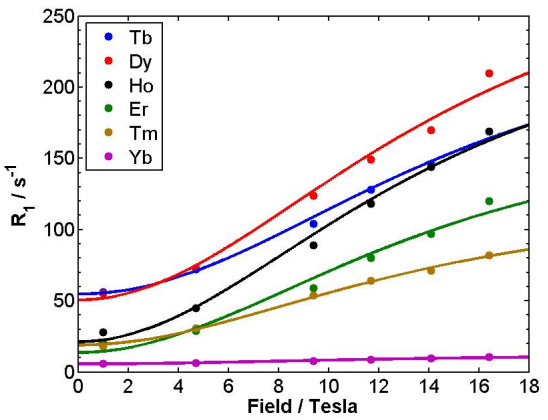

Figure 9 (left) Relaxation rates for [Ln. $\left.\mathrm{L}^{1}\right],\left(\mathrm{D}_{2} \mathrm{O} 295 \mathrm{~K}\right)$, showing best fits to eq. 1, extrapolated to zero field; (right) similar analysis for [Ln. $\left.\mathrm{L}^{2}\left(\mathrm{H}_{2} \mathrm{O}\right)\right]$ showing the large difference in behaviour for complexes of Er, Tm, Dy and Tb. ${ }^{7}$

In the azaphosphinate system (Figure 9), the $\mathrm{Er}^{3+}$ complex relaxes faster than the $\mathrm{Dy}^{3+}, \mathrm{Ho}^{3+}$ and $\mathrm{Tb}^{3+}$ analogues at all fields: such behaviour is unprecedented and can only be explained within BRW relaxation theory, by both a long $T_{1 \mathrm{E}}$ for $\operatorname{Er}(0.85 \mathrm{ps}$, 
compared to 0.17 ps for the carboxylate analogue) and an apparent $\mu_{\text {eff }}$ value that is comparable to, or larger than that for Dy and Ho, at this sizeable ligand field. Consequently, significant deviations in $\mu_{\mathrm{eff}}$ values were needed $(-11 \% \mathrm{Dy} ;+5 \% \mathrm{Er}$; $+19 \% \mathrm{Tm}$ ) from those calculated for 'free ions', using the Landé and van Vleck approximations, ${ }^{16}$ to fit the unusually fast relaxation of the Tm and Er phosphinate complexes. The $\mu_{\text {eff }}$ values are often predicted to be independent of coordination environment, ${ }^{16}$ but in systems such as the azaphosphinate series, it cannot be assumed that the ligand field is insignificant with respect to the spin-orbit coupling, as $B^{2}{ }_{0}=$ $570 \mathrm{~cm}^{-1}$, almost three times $\mathrm{k} T$ at room temperature.

Studies by Hölsä (Table 1) have also shown that ${ }_{2}$ in a series of [ $\mathrm{LnOBr}$ ] compounds that have $B_{0}^{2}$ values from $-1200(\mathrm{~Tb})$ to $-1350 \mathrm{~cm}^{-1}(\mathrm{Yb})$, the room temperature $\mu_{\mathrm{eff}}$ values (measured by SQUID magnetometry) were systematically lower than the 'freeion' calculated values for all lanthanides in the series from $\mathrm{Tb}^{3+}$ to $\mathrm{Yb}^{3+}{ }^{23}$ The largest deviation was observed for $\mathrm{HoOBr}$, for which the room temperature $\mu_{\mathrm{eff}}=9.39 \mathrm{BM}$, ($12 \%$ ), and the lower values are believed to be due to the mixing of $m_{J}$ states with lower $J$ values within the ground state.

However, in the azaphosphinate system, ${ }^{7}$ where the value of $\mu_{\mathrm{eff}}$ is determined using BRW theory, caution must also be exercised with the theory itself. The BRW theory models the dipolar interaction between the $\mathrm{Ln}^{3+}$ electron density and the nucleus of interest as a point dipole interaction, and the magnetic susceptibility of the $\mathrm{Ln}^{3+}$ ion is treated as if it were isotropic and a scalar parameter. Furthermore, the Curie term of the equation, where the fourth power dependence of the $\mu_{\text {eff }}$ value is most influential, also assumes that molecular rotation is isotropic, with a correlation time $\tau_{r}$.

Finally, the anisotropy of the static ligand field of a complex can affect the degree of anisotropy found in the magnetic susceptibility tensor, which is not accounted for in the standard SBM equation. ${ }^{57}$ The anisotropy of the magnetic susceptibility tensor has been explicitly considered theoretically, and leads to a modification of equation 4 (vide supra). The impact of the anisotropy of the magnetic susceptibility tensor on relaxation was first mooted by Vega and leads to eq. 5 , below. ${ }^{57}$ 


$$
\begin{aligned}
& R_{1}=-4 F \frac{3 \tau_{r}}{1+9 w_{N} \tau_{r}^{2}}+4 F^{1} \frac{\tau_{r}}{1+w_{N}^{2} \tau_{r}^{2}} \\
& \text { where } F=-\frac{3}{8}\left(\frac{1}{4 \pi}\right)^{2} \frac{w_{N}^{2} \varepsilon}{r^{6}} \\
& F^{1}=\frac{3}{10}\left(\frac{1}{4 \pi}\right)^{2} \frac{w_{N}^{2}}{r^{6}}\left[\chi^{2}+4 \pi r^{3} \chi \delta^{p c s}-\frac{\varepsilon}{4}+\left(\chi_{x x}^{2}+\chi_{y y}^{2}+\chi_{z z}^{2}-3 \chi^{2}\right) / 6\right] \\
& \varepsilon=\frac{x^{2} y^{2}\left(\chi_{x x}-\chi_{y y}\right)^{2}+x^{2} z^{2}\left(\chi_{x x}-\chi_{z z}\right)^{2}+y^{2} z^{2}\left(\chi_{y y}-\chi_{z z}\right)^{2}}{r^{4}}
\end{aligned}
$$

This modified treatment has yet to be used to analyse detailed relaxation data sets, but should be in the future. The barrier to doing so is the need to assess or measure the magnitude of the components of the magnetic susceptibility tensor, for which detailed susceptibility measurements are required. Undoubtedly, relaxation analyses need to take the anisotropic electron distribution and ligand field into account, especially for low symmetry systems that are often used when incorporating functionality into new MRI sensing or imaging probes.

\section{Optical behaviour: the size and anisotropy of the electric susceptibility tensor}

\section{Towards Judd-Ofelt theory}

Optical properties of lanthanides have been captivating researchers for over a century since the pioneering work of Crookes $^{58}$, who used emission spectroscopy to identify lanthanides in ores, discovering europium in 1885, sixteen years before it was isolated by Demarçay. In that era, optical spectroscopy was considered as an auxiliary tool in chemical analysis; no efforts were made to attempt spectral analysis.

For some time, it remained unclear why Laporte-forbidden $\mathrm{f}-\mathrm{f}$ transitions in lanthanides were observed, until the work of Van Vleck ${ }^{59}$. He pointed out three reasons that may be responsible for a partial breakdown of selection rules. As the Laporte selection rules are valid for electric-dipole (ED) transitions only, one of the possible explanations was the non-ED nature of the observed bands. Van Vleck suggested that they could be either quadrupole or magnetic-dipole allowed. Meanwhile, he admitted that these bands can still be mainly ED in character, but the Laporte selection rules were violated by distortion of the electronic distribution by the crystal field, either by asymmetry i.e. a static 'non-centrosymmetric' crystal field, or by molecular vibrations. 
Experimental optical studies were stimulated by the introduction of the photomultiplier tube, allowing emission spectra to be acquired with higher resolution and more precise determination of oscillator strength. The series of experiments performed in the Zeeman Laboratory were summarised by Broer ${ }^{60}$, revising earlier work of Van Vleck. He concluded that induced ED transitions are mainly responsible for the observed emission bands, whilst quadrupole-allowed transitions should be of substantially lower intensity, and magnetic dipole transitions can contribute only to a limited number of transitions 'under especially favourable circumstances'. It took another 16 years, before Judd and Ofelt independently published their work, ${ }^{10}$ where they rationalised the nature of observed induced ED transitions by admixing states of opposite parity $\left(4 f^{n-1} 5 d^{1}\right.$ and $4 f^{n-1} 5 g^{1}$ ), mediated by odd-order crystal field parameters. Thus, they connected optical properties (oscillator strength) with structural factors ('odd-order' crystal field parameters).

The proposed theory was based on several assumptions: (i) admixing levels of opposite parity were degenerate with regard to $J$; (ii) the energy difference between $\mathrm{f}$ levels was negligibly smaller than that between f-level and $4 f^{n-1} 5 d^{1}$ and $4 f^{n-1} 5 g^{1}$ levels; (iii) Stark levels of the ground state were assumed to be equally populated; (iv) the material was assumed to be optically isotropic. The final expression for the oscillator strength, $f$, of the ED transition (equation 6) depends on so-called JuddOfelt parameters $\left(\Omega_{\lambda}, \lambda=2,4,6\right)$ :

$$
f=\frac{8 \pi^{2} m c}{3 h \bar{\lambda}(2 J+1)} \mathrm{n}\left(\frac{n^{2}+2}{3 n}\right)^{2} \sum_{\lambda=2,4,6} \Omega_{\lambda}\left|\left\langle J\left\|U^{\lambda}\right\| J^{\prime}\right\rangle\right|^{2}
$$

in which $U^{\lambda}$ is a unit tensor with tabulated matrix elements, $J$ and $J^{\prime}$ are total angular momentum for ground and excited states, respectively, $\eta$ is a refractive index of the medium. Judd-Ofelt parameters $\Omega_{\lambda}$ in principle can be calculated $a b$ initio, in accordance with the following expression (eq. 7):

$$
\Omega_{\lambda}=(2 \lambda+1) \sum_{t, p}\left|A_{t p}\right|^{2} \frac{\Xi^{2}(\lambda, t)}{(2 t+1)}
$$

where $A_{t p}$ are crystal field parameters and $\Xi(\lambda, t)$ represents $4 f \rightarrow 4 f$ emission intensity in the Judd-Ofelt theory. However, these parameters are still used mainly as phenomenological parameters in semi-empirical calculations to fit the experimentally observed spectra. A major obstacle, whilst calculating $\Omega_{\lambda}$, is insufficient precision of the radial integrals (used to calculate $\Xi(\lambda, t)$ ) and the lack of precisely determined crystal field parameters $A_{t p}$. Therefore, the oscillator strengths cannot be reliably predicted from the known crystal structure. Another drawback of the initial theory is the fact that it cannot explain the origin of certain transitions (i.e. ${ }^{5} D_{0} \rightarrow{ }^{7} F_{0}$ in $\mathrm{Eu}^{3+}$ ), as well as the nature of so-called hypersensitive transitions, which are particularly sensitive to coordination environment. Moreover, this theory neglects the interaction between the lanthanide ion and surrounding ligands, even though a charge 
transfer state can significantly contribute to the emission spectra of some lanthanides. Therefore, efforts were made to modify the initial theory to address these limitations.

\section{Hypersensitive transitions}

The most discerning definition for hypersensitive transitions was given by Gruen $^{61}$ : 'for a given lanthanide ion, it often happens that one or more of the narrow absorption bands become more intense, by as much as a factor of 4 or 5 , on replacing one ligand by another, whereas the other narrow bands show almost no change in intensity'. Judd and Jørgensen noted ${ }^{62}$ that these transitions obey the selection rules for $U^{(2)}$ unit tensor $\Delta J \leq \pm 2, \Delta L \leq \pm 2, \Delta S=0$, which actually correspond to the selection rules for quadrupole transitions. Usually, the observed transitions do obey the selection rule on $\Delta J$, whilst selection rules on $\Delta L$ and $\Delta S$ are less rigid, since the transitions occur in the limit of the intermediate coupling scheme. For example, the most frequently described ${ }^{5} D_{0} \rightarrow{ }^{7} F_{2}$ transition for $\mathrm{Eu}^{3+}$ adheres to the selection rule on $\Delta J$ and $\Delta L$, but breaks the selection rule for $\Delta S$. Similar observations had already been made in the 1930s, when emission spectra of $\mathrm{Nd}^{3+}, \mathrm{Eu}^{3+}, \mathrm{Ho}^{3+}$ and $\mathrm{Er}^{3+}$ in aqueous solutions were recorded by Selwood. He had noted the significant change of relative intensity, but the lack of a coherent theory for f-f transitions left the hypersensitivity issue unresolved ${ }^{63}$. However, it was shown that predicted intensities of the electric quadrupole transitions, within classical Judd-Ofelt theory, are much smaller than experimentally observed values ${ }^{60}$. Moreover, the crystal field assumptions underlying Judd-Ofelt theory predict the reciprocal order for Judd-Ofelt parameters, depending on their sensitivity to the coordination environment $-\Omega_{6}>\Omega_{4}>\Omega_{2}$, making transitions governed by the $U^{(2)}$ unit tensor the least sensitive. ${ }^{64}$

The first attempt to explain the origin of these bands was made by Judd and Jørgensen $^{62}$ who attributed hypersensitive transitions of lanthanide ions in solution to pseudo-quadrupole transitions arising from inhomogeneity of local solvation, thereby creating an asymmetric distribution of oscillating (solvent) dipoles around the lanthanide ion. This enhances 'spatial variation of the electric vector', enhancing the intensity of a quadrupole transition. Meanwhile, they pointed out the difference between pristine quadrupole transitions and these pseudo-quadrupole bands, based on the dependence of corresponding Judd-Ofelt parameters on the wavelength. JuddOfelt parameters obtained for induced electric dipole transitions do not depend on the absorption (emission) wavelength, whilst parameters for pseudo-quadrupole transitions depend on $\lambda^{-2}$. However, this theory cannot account for the occurrence of hypersensitive transitions in crystalline materials.

Judd ${ }^{65}$ then suggested the inclusion of $Y_{1 m}$ harmonics in the Hamiltonian, to represent the interaction of the lanthanide ion with its surroundings. He listed all the point symmetry groups possessing these harmonics, $Y_{1 m}$. Such an expansion of the Hamiltonian affected only the $\Omega_{2}$ parameter, and resulted in the change of intensity of hypersensitive transitions. Thereby, Judd complemented his previous assumption ${ }^{10}$ on quadrupolar enhancement by dielectric inhomogeneity, with a simple change of point symmetry around the $\mathrm{Ln}^{3+}$ ion. Nevertheless, the latter mechanism could not 
account for hypersensitive transitions in gaseous $\mathrm{NdI}_{3}$ with $\mathrm{D}_{3 \mathrm{~h}}$ symmetry, which does not have $Y_{l m}$ harmonics, yet exhibits strong hypersensitive bands. Dielectric inhomogeneity also cannot contribute to the observed intensity of hypersensitive transitions, as the predicted intensity is lower by several orders of magnitude than the observed value. Therefore, a vibronic mechanism, previously rejected by Judd and Jørgensen for solutions of $\mathrm{Ln}^{3+}$ but found to be appropriate for lanthanide compounds in the gaseous phase, along with covalency effects was suggested by Gruen to explain the observed hypersensitivity, providing decent correspondence to the observed JuddOfelt parameters ${ }^{6,10}$. However, parameters they used for calculations (viz. radius of vibrating complex and amplitude of vibrations) were brought into question ${ }^{66}$, and the proper use of these parameters predicts the larger $\Omega_{2}$ value for $\mathrm{NdBr}_{3}$ compared to $\mathrm{NdI}_{3}$, though the reciprocal trend is experimentally observed. Peacock concluded ${ }^{67}$ that such a vibronic mechanism could significantly contribute to hypersensitive transitions only in the case of inorganic materials with strong lattice vibrations, whilst it is almost negligible for molecular complexes.

Later, an occurrence of hypersensitive transitions was also demonstrated by Blasse $e^{68}$ for solid-state samples with site symmetry $\mathrm{D}_{2 \mathrm{~d}}$, which do not contain $Y_{1 m}$ harmonics, in $\mathrm{NaGdO}_{2}-\mathrm{Eu}^{3+}$. By 'covalent effects', the hybridization of $4 \mathrm{p}$ and $4 \mathrm{f}$ orbitals was invoked to enhance the efficiency of mixing of these hybrid orbitals with $5 \mathrm{~d}$ orbitals, compared to pure $4 \mathrm{f}$ orbitals. This model, proposed by Gruen, was incomplete and was limited to heavy ligands such as bromide, whilst organic complexes with O- and $\mathrm{N}$-donor ligands were beyond its scope.

A covalency model for hypersensitive transitions was developed by Choppin ${ }^{64}$, who modified the initial procedure proposed by Judd and Ofelt by including an excited one electron charge transfer state (CT), as additional to the excited states $f^{n-1} 5 d^{1}$ and $f^{n-1} 5 g^{1}$ to be mixed with $\mathrm{f}^{\mathrm{n}}$. It was revealed that the Judd-Ofelt parameters $\Omega_{n}$, obtained in such a manner, are inversely proportional to the squared energy difference between an excited charge transfer state $\left(f^{n+1} \phi^{m-1}\right)$ and ground configuration $\left(f^{n} \phi^{m}\right)$. In turn, these energies are dependent on the optical electronegativity (eq. 8):

$$
E=30\left(X_{M}^{o p t}-X_{\text {Ligand }}^{o p t}\right)+C^{1}(n) E^{1}+C^{3}(n) E^{3}+C^{2}(n) \xi_{n f}
$$

in which $X_{M}^{o p t}$ and $X_{\text {Ligand }}^{o p t}$ are the optical electronegativities of the lanthanide ion and ligand ( $X_{\text {Ligand }}^{\text {opt }}$ is quantitatively related to the ligand $\mathrm{p} K_{a}$, leading to a correlation between oscillator strength and $\mathrm{p} K_{a}$ of the ligand), respectively, $E^{1}$ and $E^{3}$ are Racah's parameters of interelectron repulsion, $\xi_{n f}$ is the spin-orbit coupling constant and $C^{n}$ are tabulated constants. Thereby, the lower the extent of covalency, the higher the energy of the excited charge transfer state, and hence the lower the intensity of the hypersensitive transition. Trivalent europium complexes exhibit the strongest hypersensitive transition among the lanthanide series, due to the lower reduction potential of the $\mathrm{Eu}(\mathrm{III}) / \mathrm{Eu}(\mathrm{II})$ couple, which varies from $-0.35 \mathrm{~V}$ for the aqua ion to around $-1.1 \mathrm{~V}$ for typical coordination complexes with anionic ligands that stabilise 
the Eu(III) state. ${ }^{69}$ The same findings were reported in an expanded covalency approach by Blasse $^{70}$ for lanthanide-containing compounds, arriving at the conclusion that mixing of $f^{n}$ states with $f^{n-1} 5 d^{1}$ and $f^{n-1} 5 g^{1}$ is less important than mixing with the charge transfer state for Eu(III) compounds, because $f^{n-1} 5 d^{1}$ and $f^{n-1} 5 g^{1}$ have substantially higher energies than the CT state.

\section{Dynamic coupling mechanism}

The original Judd-Ofelt theory (referred to as 'static coupling' - SC) considers the perturbation of the wavefunction of the $\mathrm{Ln}^{3+}$ ion by the ligand field, but neglects the reciprocal influence of metal wavefunctions on the ligand wavefunction. The charge distribution around the lanthanide ion induces dipoles on the ligands, and the correlation of the quadrupole (more generally, multipole) on the $\mathrm{Ln}^{3+}$ ion with an

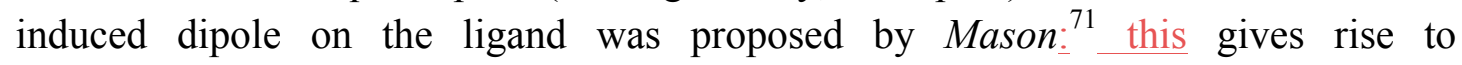
hypersensitive transitions because this mechanism contributes only to $\Omega_{2}$. The expression for the oscillator strength of an $\mathrm{f}-\mathrm{f}$ transition derived in accordance with this mechanism, called the 'dynamic coupling model' (DC), is analogous to that derived within the SC model, but with a different Judd-Ofelt parameter $\Omega_{2}$ (dyn) (eqs. 9 and 10):

$$
\begin{gathered}
f(\text { dyn })=\frac{8 \pi^{2} m c}{3 h \bar{\lambda}(2 J+1)} \mathrm{n}\left(\frac{n^{2}+2}{3 n}\right)^{2} \Omega_{2}(\text { dyn })\left|\left\langle J\left\|U^{2}\right\| J^{\prime}\right\rangle\right|^{2} \\
\Omega_{2}(\text { dyn })=\frac{28}{5}\left\langle 4 f\left|r^{2}\right| 4 f\right\rangle^{2} \sum_{m=0}^{3}\left(2-\delta_{m, 0}\right) \sum_{L}\left|R_{L}^{-4} \bar{\alpha}(L) C_{(3)}^{m}(L)\right|^{2}
\end{gathered}
$$

in which $R_{L}$ is the distance between the lanthanide ion and the ligand, $\bar{\alpha}(L)$ is the mean polarisability of the ligand at the transition frequency of f-electron, $v_{0 a}$ and $C_{(3)}^{m}$ are tensor components, containing angular structural information.

The theory predicts that hypersensitive transitions can be observed, if the symmetry point group contains $Y_{3 m}$ harmonics. Compared to the aforementioned assumption of $J u d d^{10}$, that the point group should contain $Y_{l m}$ harmonics, inclusion of $Y_{3 m}$ harmonics extends the number of available point groups, including the aforementioned $D_{3 h}$ group, which is present in gaseous trihalides exhibiting a hypersensitive transition (vide supra). This model was further developed by Kuroda ${ }^{12,72}$, by including the anisotropic polarisabilities of the ligands. She considered two model complexes, (Figure 10) where the point symmetry of the $\mathrm{Ln}^{3+}$ ion can be approximated as a $\mathrm{D}_{3}$ group $-\left[\mathrm{Eu}\left(\mathrm{H}_{2} \mathrm{O}\right)_{9}\right]^{3+}$ and $\left[\mathrm{Eu}(\text { oda })_{3}\right]^{3-}($ oda $=$ oxydiacetate $)$, dividing a contribution from the two distinct ligand sets, $\left[\mathrm{LnO}_{3}\right]$ and $\left[\mathrm{LnO} O_{6}\right]$, to electric dipole transition moments. It was found that the $M= \pm 1$ components of the ${ }^{7} F_{0} \rightarrow{ }^{5} D_{2}$ transition in the absorption spectra are present only 'for anisotropic ligands and only for a reduction from $\mathrm{D}_{3 \mathrm{~h}}$ in the symmetry of one or both of the ligand sets'. Therefore, this transition was observed for $\left[\mathrm{Eu}(\mathrm{oda})_{3}\right]^{3-}$ with a distortion to $\mathrm{D}_{3}$ symmetry in the $\left[\mathrm{EuL}_{6}\right]$ ligand set, but was absent in the case of $\left[\mathrm{Eu}\left(\mathrm{H}_{2} \mathrm{O}\right)_{9}\right]^{3+}$ with an effective $\mathrm{D}_{3 \mathrm{~h}}$ 
symmetry in each ligand set. Even slight distortions from ideal symmetry can significantly change the mean polarisability of the ligand $\bar{\alpha}(L)$, and hence the intensity of the hypersensitive transition. Rotation of the $\left[\mathrm{EuO}_{3}\right]$ ligand set by $5^{\circ}$ relative to the $\left[\mathrm{Eu} O_{6}\right]$ set, transforms a trigonal prism into a tricapped trigonal prism and leads to an order of magnitude increase of $\bar{\alpha}(L)$.
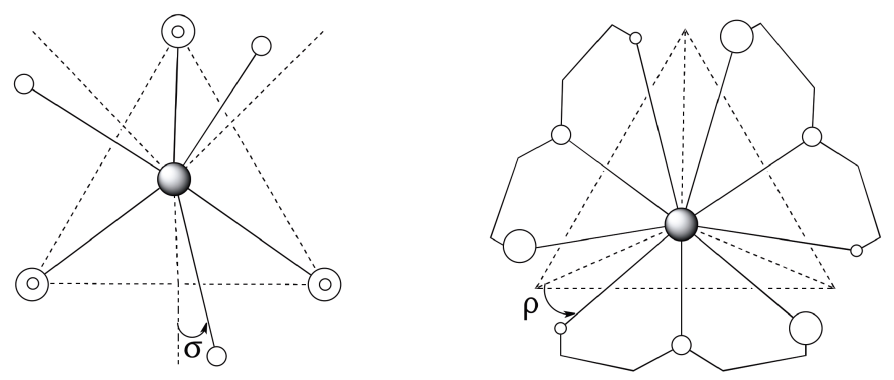

Figure 10 Projections on the plane perpendicular to the trigonal axis of the lanthanide complex ion $\left[\mathrm{Eu}\left(\mathrm{H}_{2} \mathrm{O}\right)_{9}\right]^{3+}($ left $)$ and $\left[\mathrm{Eu}(\mathrm{oda})_{3}\right]^{3-}($ right $)$. Angular distortions are designated $\sigma$ and $\rho$, respectively. ${ }^{72}$

In spite of the appearance and successful application of the DC mechanism, the SC mechanism was not completely rejected, and each mechanism was assumed to contribute to the overall intensity of hypersensitive transitions.

The first attempt to combine both approaches was made by Richardson, examining nine-coordinate lanthanide complexes in trigonal symmetry ${ }^{73}$. The approach was further developed to include the 'cross-term contributions' representing the interference between SC and DC mechanisms ${ }^{74}$. The relative contribution of each mechanism was analysed by $\operatorname{Mason}^{75}$, for the eight-coordinate tetrakis(diethyldithiocarbamate) lanthanide complexes $\mathrm{Na}\left[\operatorname{Ln}\left(\mathrm{Et}_{2} \mathrm{dtc}\right)_{4}\right]$, (Figure 11).

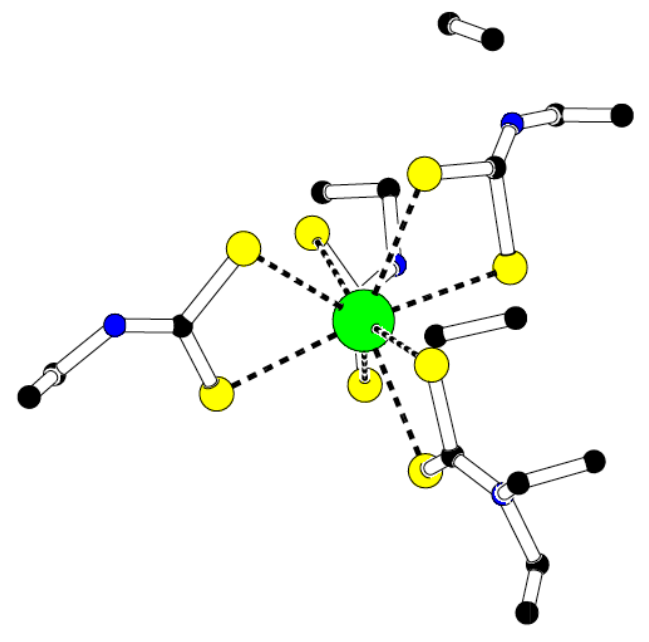

Figure 11 Molecular structure of $\mathrm{Na}\left[\mathrm{La}\left(\mathrm{Et}_{2} \mathrm{dtc}\right)_{4}\right]^{76}$ (owing to disorder, some Et groups appear not to be bound to N) .

The overall Judd-Ofelt parameter (eq. 11) was made up of three components, $\Omega_{\lambda}(C F)$ (crystal field contribution from SC model), $\Omega_{\lambda}(L P)$ (ligand polarisation contribution 
from DC model) and $\Omega_{\lambda}(C T)$, the cross-term contribution representing the scalar product of the crystal field and ligand field polarisation components:

$$
\Omega_{\lambda}(t o t)=\Omega_{\lambda}(C F)+\Omega_{\lambda}(L P)+\Omega_{\lambda}(C T)
$$

It was shown that when the relative contribution of $\Omega_{\lambda}(L P)$ was larger for $\Omega_{2}(66 \%)$, than for $\Omega_{4}(17 \%)$ and $\Omega_{6}(0.1 \%)$, then DC was the determining mechanism responsible for hypersensitivity. A close relative distribution between these three components was observed across the whole lanthanide row from $\operatorname{Pr}(\mathrm{III})$ to $\mathrm{Yb}(\mathrm{III})$. The ratio $\frac{\Omega_{2}(L P)}{\Omega_{2}(C F)}$ for $\mathrm{Na}\left[\mathrm{La}\left(\mathrm{Et}_{2} \mathrm{dtc}\right)_{4}\right]$ was found to be larger than for the aforementioned systems, $\left[\mathrm{Eu}\left(\mathrm{H}_{2} \mathrm{O}\right)_{6}\right]^{3+}$ and $\left[\mathrm{Eu}(\mathrm{oda})_{3}\right]^{3-}$, owing to the higher polarisability of sulphur and the presence of a different polyhedron around the lanthanide ion (distorted tetrahedral vs. trigonal prism), providing an optimal angular term in the tensors $C_{(3)}^{m}(L)$ for the case of a $\left[\mathrm{LnO}_{8}\right]$ set, compared to mutually interfering out-of-phase sets for the trigonal planar $\left[\mathrm{LnL}_{3}\right]$ and trigonal prismatic $\left[\mathrm{LnL}_{6}\right]$ moieties in the $\left[\mathrm{LnO}_{9}\right]$ polyhedron. Subsequently, this dual approach was extended to calculations of MD transition and rotatory strength of chiral lanthanide complexes. ${ }^{77}$

\section{Selected example of spectral analysis}

Notwithstanding the rich theoretical background that seeks to explain the origin of $\mathrm{f}-\mathrm{f}$ transitions in lanthanide complexes, there are very few examples employing spectroscopic analysis that are based on a combined SC and DC approach. A recent example from Di Bari ${ }^{78}$, used Richardson's approach ${ }^{74}$ and was employed to predict the electronic circular dichroism spectrum of a chiral $\mathrm{Yb}(\mathrm{III})$ complex.

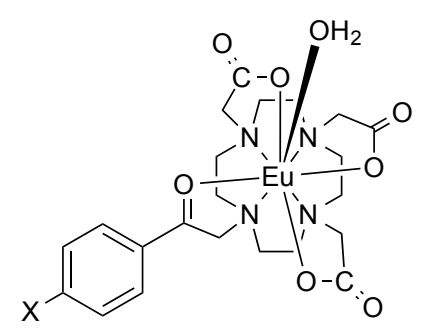

Figure 12 Structure of europium phenacyl-DO3A complexes $\left(\mathrm{X}=\mathrm{H}, \mathrm{OMe} \text { or } \mathrm{NMe}_{2}\right)^{79}$

An incorrect description of the factors that give rise to hypersensitivity can still be encountered. ${ }^{80}$ Typically, the high intensity of hypersensitive transition is attributed only to the low symmetry of the coordination site (SC model), ignoring the polarisability of the ligating atoms (DC model). To evaluate the influence of polarisability on the intensity of hypersensitive transitions, it is necessary to exclude any influence of site symmetry, i.e. the coordination polyhedron around $\mathrm{Eu}^{3+}$ should be preserved, when changing substituents with different polarisabilities. Macrocyclic $12-\mathrm{N}_{4}$ (and 9- $\mathrm{N}_{3}$ ) ligand platforms can be considered as an appropriate choice, 
because the tetra-substituted $\mathrm{N}_{4}$ moiety provides a rigid coordination environment around the lanthanide ion. The influence of para-substituents on the relative intensity of the ${ }^{5} D_{0} \rightarrow{ }^{7} F_{2}$ transition in a Eu-DO3A derivative (DO3A is 1,4,7,10tetraazacyclododecane-triacetate) with a coordinated acetophenone sensitising group has been studied (Figure 12) ${ }^{79}$. By increasing the electron releasing ability of the para-substituent, the polarisability of the keto-carbonyl group was enhanced, resulting in an increase of the relative intensity of the ${ }^{5} D_{0} \rightarrow{ }^{7} F_{2}$ transition $\frac{{ }^{5} D_{0} \rightarrow{ }^{7} F_{2}}{{ }^{5} D_{0} \rightarrow{ }^{7} F_{1}}$, from 0.67 for the parent complex to 1.4 and 3.2 for $p$-OMe and $p-\mathrm{NMe}_{2}$ derivatives, respectively. It is likely that the observed changes may have been much larger, had the carbonyl oxygen occupied the capping axial position, in the mono-capped squareantiprismatic coordination.

Related observations have been made for a series of nine-coordinate europium complexes with oxy-diacetate, $\left(\left[\mathrm{Eu}(\mathrm{ODA})_{3}\right]^{3-}\right)$, imino-diacetate, $\left(\left[\mathrm{Eu}(\mathrm{IDA})_{3}\right]^{3-}\right)$, pyridine-2,6-dicarboxylate, $\left(\left[\mathrm{Eu}(\mathrm{DPA})_{3}\right]^{3-}\right)$ and a conjugated pyridyl-alkynyl ligand complex, $\left[\mathrm{Eu} . \mathrm{L}^{1}\right]$ (Figure 13). Each complex is expected to possess approximate $\mathrm{C}_{3}$ symmetry in solution. The relative intensity of the ${ }^{5} D_{0} \rightarrow{ }^{7} F_{2}$ transition ( $v s$ the $\Delta J=$ 1 manifold) increases from 1.9 (hard neutral oxygen donor) for [Eu(ODA) $]_{3}^{3-}$ to 11.1 (polarisable $\mathrm{N}_{\mathrm{py}}$ ) in [Eu. $\left.\mathrm{L}^{1}\right]$, whilst the $\Delta J=4$ transition relative intensity does not vary significantly. ${ }^{81}$
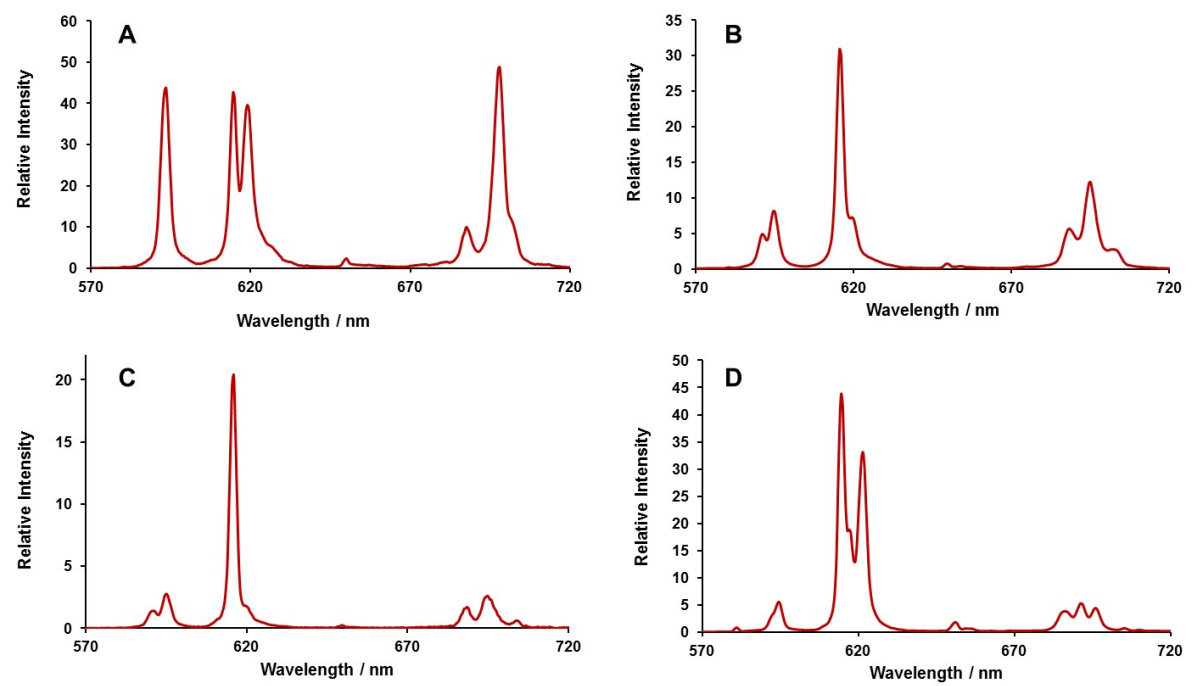

Figure 13 Comparison of total emission spectra for Eu(III) complexes showing the variation in relative emission intensity with ligand donor polarisability for these systems in approximate $\mathrm{C}_{3}$ symmetry.: (A) [Eu(ODA $\left.\left.)_{3}\right]^{3-}\left(\lambda_{\text {exc }}=395 \mathrm{~nm}, \mathrm{H}_{2} \mathrm{O}, \mathrm{pH} \text { 8.5); (B) [Eu(IDA) }\right)_{3}\right]^{3-}\left(\lambda_{\text {exc }}=395 \mathrm{~nm} \mathrm{H}_{2} \mathrm{O}\right.$, pH 8.5); (C) [Eu(DPA) $]_{3}{ }^{3-}\left(\lambda_{\text {exc }}=395 \mathrm{~nm}, \mathrm{pH} 8.5\right)$; (D) [Eu.L1] $\left(\lambda_{\text {exc }}=332 \mathrm{~nm}, \mathrm{pH} 7.4\right)$; (ODA oxydiacetate; IDA- iminodiacetate; DPA - pyridine-2,6-dicarboxylate). ${ }^{81}$

[Eu. $\left.\mathrm{L}^{1}\right]$ 


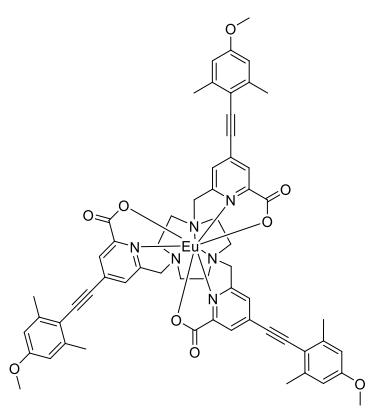

A related example ${ }^{82}$ has examined different $\mathrm{Eu}(\mathrm{III})$ complexes with both dibenzoylmethonate and various sulfoxide ligands (Figure 14). Neglecting differences in their site symmetry, the relative intensity of the hypersensitive transition increased on going from the least polarisable dialkylsulfoxide to the most polarisable diarylsulfoxide derivative.
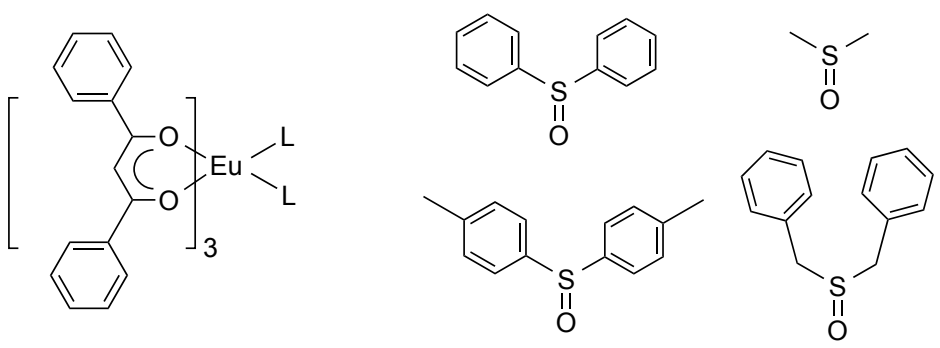

Figure 14 Structures of $\left[\mathrm{Eu}(\mathrm{dbm})_{3} \mathrm{~L}_{2}\right]$ complexes, where L $=$ DMSO, PTSO, DPSO, DBSO ${ }^{82}$

An extensively developed Sparkle model ${ }^{83}$ has been introduced, based on a very simple assumption. The $\mathrm{Ln}^{3+}$ ion is considered as a point charge - 'Sparkle', which decays with a distance as a function of $e^{-\alpha R}$, where $\alpha$ corresponds to the size of the ion. It has proved to be useful in qualitatively predicting spectra for different lanthanide-containing species. Its accuracy compares favourably to more complex $a b$ initio DFT calculations, yet requires considerably less computational time. ${ }^{84}$

A recently released Lumpac package ${ }^{85}$ allows structural optimization of a lanthanide complex calculated using the Sparkle model by varying the positions of ligating atoms to get the best fit between calculated and experimentally observed Judd-Ofelt parameters. The Judd-Ofelt parameters were calculated within a Simple Overlap Model (SOM), developed by Malta ${ }^{86}$ that assumes the potential energy of the $4 \mathrm{f}$ electrons is produced by uniformly distributed charges over small regions, centred at the mid-point between the $\mathrm{Ln}^{3+}$ ion and the ligand, and that the total charge in each region - ge $\rho$, where $\rho$ is proportional to the magnitude of the total orbital overlap between the lanthanide and the ligand. Such an assumption drastically simplifies the calculation of ligand-field parameters $\gamma_{p}^{t}$ and $\Gamma_{p}^{t}$ for SC and DC contributions, respectively, (eq. 12) which are altered to attain the lowest value $F$ : 


$$
F=\left(\Omega_{2}^{\text {exp. }}-\Omega_{2}^{\text {calc. }}\right)^{2}+\left(\Omega_{4}^{\text {exp. }}-\Omega_{4}^{\text {calc }}\right)^{2}
$$

However, the proposed approach, as with many other semi-empirical calculations, lacks any predictive ability. Improved methods are needed that allow more accurate predictions of relative intensities, especially of hypersensitive transitions, in the luminescence spectra of lanthanide complexes.

\section{Conclusions and Outlook}

The work described in this review provides a body of evidence that defines the need for more effective theories that can predict the behaviour of open shell lanthanide complexes. Researchers in the field of molecular magnetism have already considered the nature of anisotropy in lanthanide ground state manifolds, and are using the predictive capabilities of existing theoretical approaches to suggest coordination geometries that might give rise to high temperature magnetic hysteresis. ${ }^{15}$ However, the luminescence and magnetic resonance properties of even simple complexes are complicated both by the nature of the ground state manifold and by thermal population of the higher $m_{J}$ Stark levels. The relative energies of these levels can be altered, and even reversed by the nature of the ligand field - giving rise to significant changes in the overall magnetic anisotropy. If such systems can be understood in detail, it will become possible to design and control the degree of anisotropy. At the moment, the observed spectra of a range of complexes give some indication of the scope of what can be achieved, and the work of Yamashita and Enders has signposted the way forward in detailed NMR analyses of systems, such as terbium phthalocyanato complexes, whose magnetic susceptibility behaviour has been thoroughly studied, driven by their unique 'single molecule magnet' properties. ${ }^{15}$

However, planned rather than serendipitous progress must await the development of effective predictive theories that define the magnetic and/or electric susceptibility tensors in lanthanide containing systems. Achieving such a goal would facilitate a leap forward in the design of a wide variety of systems, including contrast media and EPR relaxation agents, in which minimizing the crystal field parameters will minimize the anisotropy at the metal centre, or controlling the chemical shift range in molecular systems.

In MRI and MR spectral imaging techniques, the nature of any observed pseudocontact shift is highly dependent on the local coordination environment and directly reflects the anisotropy at the lanthanide centre. If this can be fully exploited, it may be possible to take a new approach to responsive imaging- relying less upon the development of complexes that offer selectivity for one analyte over another through binding, and instead exploit the different effects of a range of analytes in a single system. Such an approach would require relatively slow exchange of analytes on the NMR timescale, but would have the merit of allowing direct multiplexingprovided that the anisotropy can be tuned between different species to the point that 
signals are clearly separated. Other obvious approaches include permuting the lanthanide ion in complexes of a common ligand to generate distinctive shift behaviour, that then allows the temperature and local environment effects to be distinguished, e.g. for $\mathrm{pH}$ or $\mathrm{pM}$ responsive probes in vivo.

The same changes to pseudocontact shift with ligand field also mean that caution must be exercised in the design of structural probes that exploit lanthanide complexes. It is clear that any system used as a spin probe or PCS probe of structure must have an invariant local crystal field that cannot easily be influenced by local donor atoms i.e. the metal must be shielded completely from its environment, while the nature of the ligand should not be influenced significantly by its surroundings. It is far from clear that these criteria are met by the current generation of structural probes that are used in protein analysis, for example. ${ }^{87}$

In the field of optical spectroscopy, there are even greater uncertainties. For a start, while NMR methods immediately reveal the nature of the anisotropy in a system, optical methods only give a clear idea of the magnitude of anisotropy through study of the fine structure of a transition- they do not invariably give a clear picture of the dominant magnetic axis. Still less do current theories allow effective prediction of non-linear optical properties.

Bleaney's theories of magnetism have provided the basis for discussing the behaviour of lanthanide containing complexes over the course of nearly half a century. However, they are based upon a series of approximations that do not hold up well in many systems. In particular, the goodness of $J$ as a quantum number is far from certain, while the impact of higher order ligand field terms was given insufficient weight. The challenge remains to build a better approach, and modern computational methods offer an ideal opportunity to do so.

As in so many other fields, structure and spectral properties are inextricably linked. When it comes to the spectroscopy of lanthanide complexes, the challenge that must be addressed lies in demonstrating and establishing the nature of that link. Such an approach can be used to rationalise the non-linear optical behaviour of compounds and materials. Therefore, the anisotropy of ligand dipolar polarisability will play a key role in rationalising emission intensity changes, which are often critical in monitoring a change in the lanthanide coordination environment.

More ambitiously, and thinking back to Maxwell's outstanding contributions to electromagnetism in the nineteenth century, a combined theory could be contemplated, examining electric and magnetic susceptibility tensors and their interaction with lanthanide (III) systems, in the appropriate electromagnetic radiation field. 
Acknowledgements We thank the ERC (266804 and 267426), and EPSRC (EP/N006909/1) for support.

\section{References}

1. J-C. G. Bunzli, Chem.Rev. 2010, 110, 2729.

2. M. C. Heffern, L. M. Matosziuk and T. J. Meade, Chem. Rev. 2014, 114, 4496.

3. J. M. Zwier, H. Bazin, L Lamarque, G. Mathis, Inorg. Chem. 2014, 53, 1854.

4. C. Piguet and C. F. G. C. Geraldes, Handbook on the Physics and Chemistry of Rare Earths, K. A. Gschneidner and L. Eyring, eds. Elsevier, 2003, 353-463.

5. I. Bertini, C. Luchinat and G. Parigi, Prog. Nucl. Magn. Reson. Spectrosc. 2002, 40, 249.

6. A. M. Funk, K-L. N. A. Finney, P. Harvey, A. M. Kenwright, E. R. Neil, N. J. Rogers, P. K. Senanayake and D. Parker, Chem. Sci. 2015, 6, 1655.

7. A. M. Funk, P. Harvey, K-L. N. A. Finney, M. A. Fox, A. M. Kenwright, N. J. Rogers, P. K. Senanayake, D. Parker, Phys. Chem. Chem. Phys. 2015, 17, 16507; N. J. Rogers, K-L. N. A. Finney, P. K. Senanayake and D. Parker, Phys. Chem. Chem. Phys. 2016, 18, in press, DOI: 10.1039/C5CP06755C.

8. O. A. Blackburn, N. F. Chilton, K. Keller, C. E. Tait, W. K. Myers, E. J. McInnes, A. M. Kenwright, P. D. Beer, C. R. Timmel, S. Faulkner, Angew. Chem. Int. Ed. Engl. 2015, 54, 10783.

9. O. A. Blackburn, A. M. Kenwright, P. D. Beer and S. Faulkner, Dalton Trans. 2015, 44, 19509; O.A. Blackburn, J.D. Routledge, L.B. Jennings, N.H. Rees, A.M. Kenwright, P.D. Beer and S. Faulkner, Dalton Trans. 2016, DOI: 10.1039/ c5 dt04349b

10. G. S. Ofelt, J. Chem. Phys. 1962, 37, 511; B. R. Judd, Phys. Rev. 1962, 127, 750.

11. S. F. Mason S.F., R. D. Peacock, and B. Stewart, Mol. Phys. 1975, 30, 1829.

12. R. Kuroda, S. F. Mason and C. Rosini, Chem. Phys. Lett. 1980, 70, 11; S. F. Mason, Struct. Bonding (Berlin), 1980, 39, 43.

13. M. F. Reid and F. S. Richardson, Chem Phys Lett. 1983, 95, 501.

14. R. G. Denning, J. Harmer, J. C. Green, M. Irwin, J. Am. Chem. Soc., 2011, 133, 20644.

15. J. D. Rinehart and J. R. Long, Chem. Sci. 2011, 2, 2078; N. Ishikawa, M. Sugita, T. Ishikawa, S. Koshihara, J. Phys. Chem. B 2004, 14, 11265; M. Damjanovic, K. Katoh, M. Yamashita, M Enders, J. Am. Chem. Soc. 2013, 135, 14349; M. Dmajanovic, T. Morita, K. Katoh, M. Enders, Chem. Eur-J. 2015, 21, 14421.

16. J. Jensen, A. R. Mackintosh, Rare Earth Magnetism; International Series Monographs of Physics; Clarendon Press: Oxford, 1991; H. Curzen, L. Bovigny, C. Bulloni, C. Daul, Chem. Phys. Lett. 2013, 574, 129.

17. A. S. Souza, M. A. Couto dos Santos, Chem. Phys. Lett. 2012, 521, 138; V. S. Mironov, Y. G. Galyametdinov, A. Ceulemans, C. Görller-Walrand, K. Binnemans, J. Chem. Phys. 2002, 116, 4673.

18. C. Görller-Walrand and K. Binnemans in Handbook on the Physics and Chemistry of Rare Earths; K. A. Gschneidner and L. Eyring, eds. Elsevier, 1996, 23, 121.

19. C-G. Ma, M. G. Brik, V. Kiisk, T. Kangur, I. J. Sildos, J. Alloys Compd. 2011, 509, 3441; F. Auzel, O. L. Malta, J. Phys. 1983, 44, 201; O. L. Malta, E. AnticFidancev, M. Lemaitre-Blaise, A. Milicic-Tang, M. Taibi, J. Alloys Compd. 1995, 228, 41.

20. J. Sievers, Z. Phys. B Con. Mat. 1982, 45, 289. 
21. N. F. Chilton, C. A. P. Goodwin, D. P. Mills and R. E. P. Winpenny, Chem. Commun. 2015, 51, 101.

22. G. Cucinotta, M. Perfetti, J. Luzon, M. Etienne, P-E. Car, A. Caneschi, G. Calvez, K. Bernot, R. Sessoli, Angew. Chem. Int. Ed. Engl. 2012, 51, 1606.

23. J. Hölsä, M. Lastusaari, J. Niittykoski, R. Sàez Puche, Phys. Chem. Chem. Phys. 2002, 4, 3091.

24. B. Bleaney, J. Magn. Reson. 1972, 26, 91.

25. J. Autsbach, Ann. Rep. Comput. Chem. 2015, 11, 3-36; F. Genrdon, K. Sharkas, J. Autsbach, J. Phys. Chem. Lett. 2015, 6, 2183.

26. A. Soncini, W. Van den Heuwel, J. Chem. Phys. 2013, 138, 021103; W. Van den Heuwel, A. Soncini, J. Chem. Phys. 2013, 138, 154113

27. B. R. McGarvey, J. Magn. Reson. 1979, 33, 445; E. W. Stout, H. S. Gutowsky, J. Magn. Reson. 1976, 24, 389.

28. R. Golding and P. Pyykkö, Mol. Phys. 1973, 26, 1389.

29. V. Mironov, Y. Galyametdinov, A. Ceulemans, Chem. Phys. Lett. 2001, 345, 132.

30. V. S. Mironov, Y. G. Galyametdinov, A. Ceulemans, C. Görller-Walrand, and K. Binnemans, J. Chem. Phys., 2002, 116, 4673.

31. C. M. Dobson, B. A. Levine, R. B. Martin, R. J. P. Williams, and A. V. Xavier, J. Chem. Soc., Chem. Commun.1972, 791.

32. G. T. P. Charnock and I. Kuprov, Phys. Chem. Chem. Phys. 2014, 16, 20184.

33. C. Gorller-Walrand, E. Huygen, K. Binnemans, L. Fluyt, J. Phys. Cond. Matter, 1994, 7797.

34. C. Schmitz, R. Vernon, G. Otting, D. Baker, T. Huber, J. Mol. Biol. 2012, 668.

35. J. Ren, A. D. Sherry, J. Magn. Reso., Ser. B 1996, 111, 178.

36. S. Rigault, C. Piguet, J-C. G. Bünzli, J. Chem. Soc., Dalton Trans. 2000, 2045.

37. J. H. Forsberg, R. M. Delaney, Q. Zhao, G. Harakas, R. Chandran, Inorg Chem 1995, 34, 3705.

38. J. Reuben, J. Magn. Reson. 1982, 50, 233; C. Platas-Iglesias, F. Avecilla, A de Blas, C. F. G. C. Geraldes, T. Rodríguez-Blas, H. Adams, J. Mahía, Inorg. Chem. 1999, 38, 3190.

39. B. M. Alsaadi, F. J. C. Rossotti, R. J. P. Williams, J. Chem. Soc., Dalton Trans. 1980, 2147.

40. J. H. Forsberg, R. M. Delaney, Q. Zhao, G. Harakas, R. Chandran, Inorg. Chem. 1995, 34, 3705.

41. C. F. G. C. Geraldes, A. D. Sherry, G. E. Kiefer, J. Magn. Reson. 1992, 97, 290.

42. R. S. Dickins, D. Parker, J. I. Bruce, D. J. Tozer, Dalton Trans. 2003, 1264.

43. L. Di Bari, G. Pintacuda, R. S. Dickins, D. Parker and P. Salvadori, J. Am. Chem. Soc., 2000, 122, 9257.

44. R. Schmidt, N. Nippe, K. Strobel, M. Masthoff, O. Reifschneider, D. D. Castelli, C. Höltke, S. Aime, U. Karst, C. Sunderkötter, C. Bremer, C. Faber, Radiology 2014, 272, 785; E. De Luca, P. Harvey, K. H. Chalmers, A. Mishra, P. K. Senanayake, J. I. Wilson, M. Botta, M. Fekete, A. M. Blamire, D. Parker, J. Biol. Inorg. Chem. 2014, 19, 215.

45. G. Rancan, D. Delli Castelli, S. Aime, Magn. Reson. Med. 2015 in press.

46. L. P. Hwang, J. H. Freed, J. Chem. Phys. 1975, 63, 4017.

47. A. G. Redfield, IBM Journal of Research and Development 1957, 1, 19.

48. J. H. Freed, G. V. Bruno, C. F. Polnaszek, J.Phys. Chem. 1971, 75, 3385. 
49. J. W. Walton, R. Carr, N. H. Evans, A. M. Funk, A. M. Kenwright, D. Parker, D. S. Yufit, M. Botta, S. De Pinto, K-L. Wong, Inorg. Chem. 2012, 51, 8042.

50. A. M. Funk, P. H. Fries, P. Harvey, A. M. Kenwright, D. Parker, J.Phys. Chem. A 2013, 117, 905.

51. N. Bloembergen and L. O. Morgan, J. Chem. Phys. 1961, 34, 842.

52. N. Bloembergen, J.Chem. Phys. 1957, $27,572$.

53. I. Solomon, Physical Review 1955, $99,559$.

54. M. Goldman, J. Mag. Reson. 2001, 149, 160.

55. P. H. Fries and E. Belorizky, J. Chem. Phys. 2012, 136, 074513.

56. S. Aime, L. Barbero, M. Botta, G. Ermondi, J.Chem. Soc.,, Dalton Trans. 1992, 225-228.

57. A. J. Vega and D. Fiat, Mol. Phys. 1976, 31, 347.

58. W. Crookes, Proc. R. Soc. A Math. Phys. Eng. Sci. 76, 1905, 411.

59. J. H. Vleck, J. Phys. Chem. 1937, 41, 67.

60. L. J. F. Broer, C. J. Gorter, and J. Hoogschagen Physica 1945, 11, 231.

61. D. M. Gruen, J. Chem. Phys. 1966, 45, 455.

62. C. K. Jørgensen and B. R. Judd, Mol. Phys. 1964, 8, 281.

63. P. W. Selwood, J. Am. Chem. Soc. 1930, 52, 4308.

64. D. E. Henrie, R. L. Fellows and G. R. Choppin, Coord. Chem. Rev. 1976, 18, 199.

65. B. R. Judd, J. Chem. Phys. 1966, 44, 839.

66. R. D. Peacock, J. Chem. Soc. Faraday Trans. 2 1972, 68, 169.

67. R. D. Peacock, Struct. Bond. (Berlin), 1975, 22, 83.

68. G. Blasse and A. Bril, Solid State Commun. 1966, 4, 373.

69. G. Milazzo, C. Sergio and V. K. Sharma, Tables of Standard Electrode Potentials. (Wiley, 1978).

70. G. Blasse, Structure and Bonding (Berlin), 1976, 26, 43.

71. S. F. Mason, R. D. Peacock and B. Stewart, Chem. Phys. Lett. 1974, 29, 149.

72. R. Kuroda, S. F. Mason and C. Rosini, J. Chem. Soc. Faraday Trans. 2 1981, 77, 2125 .

73. F. S. Richardson, J. D. Saxe, S. A. Davis, and T. R. Faulkner, Mol. Phys.1981, 42, 1401

74. F. S. Richardson, Chem. Phys. Lett. 1982, 86, 47.

75. S. F. Mason and G. E. Tranter, Chem. Phys. Lett. 1983, 94, 29.

76. M. Ciampolini, N. Nardi, P. Colamarino and P. Orioli, J. Chem. Soc. Dalton. Trans. $1977,379$.

77. F. S. Richardson, M. T. Berry, and M. F. Reid, Mol. Phys. 1986, 58, 929.

78. R. Berardozzi and L. Di Bari, ChemPhysChem 2015, 16, 2868.

79. A. Beeby, L. M. Bushby, D. Maffeo, and J. A. G. Williams, J. Chem. Soc. Dalton Trans. 2002, 48.

80. P. A. Tanner, Chem. Soc. Rev. 2013, 42, 5090. 
81. S. J. Butler, M. Delbianco, L. Lamarque, B. K. McMahon, E. R. Neil, R. Pal, D. Parker, J. W. Walton and J. Zwier, Dalton Trans., 2015, 44, 4791.

82. E. Niyama, H. F. Brito, M. Cremona, E. E. Teotonio, R. Reyes, G. E. Brito, M. C. Felinto, Spectrochim. Acta Part A Mol. Biomol. Spectrosc. 2005, 61, 2643.

83. A. V. M. De Andrade, N. B. da Costa, A. M. Simas, and G. F. de Sá, Chem. Phys. Lett. 1994, 227, 349.

84. D. A. Rodrigues, N. B. da Costa, and R. O. Freire, J. Chem. Inf. Model. 2011, 51, 45.

85. J. D. L. Dutra, T. D. Bispo, and R. O. Freire, J. Comput. Chem. 2014, 35, 772.

86. O. L. Malta, Phys. Lett. 1982, 87, 27.

87. W-M Liu, P. H. J. Keizers, M. A. S. Hass, A. Blok, A. J. C. Saris, M. Overhand, M. Ubbink J. Am. Chem. Soc., 2012, 134, 17306. 